\title{
Gradhiva
}

\section{Le musée comme lieu d'administration de la preuve. Genèse et destin de deux collections du XIX e siècle}

Museums as a place to produce evidence: the origin and destiny of two 19th century collections

Sophie A. de Beaune

\section{OpenEdition}

Journals

Édition électronique

URL : http://journals.openedition.org/gradhiva/2741

DOI : $10.4000 /$ gradhiva.2741

ISSN : 1760-849X

Éditeur

Musée du quai Branly Jacques Chirac

Édition imprimée

Date de publication : 1 décembre 2013

Pagination : 166-199

ISBN : 978-2-35744-072-2

ISSN : 0764-8928

\section{Référence électronique}

Sophie A. de Beaune, «Le musée comme lieu d'administration de la preuve. Genèse et destin de deux collections du XIXe siècle », Gradhiva [En ligne], 18 | 2013, mis en ligne le 01 décembre 2016, consulté le 19 avril 2019. URL : http://journals.openedition.org/gradhiva/2741 ; DOI : 10.4000/gradhiva.2741 


\section{Le musée comme lieu d'administration de la preuve}

Genèse et destin de deux collections du xIx ${ }^{e}$ siècle

par Sophie A. de Beaune

Augustus Lane Fox Pitt-Rivers (1827-1900)

et Édouard Piette (1827-1906) firent tous

deux don de leur collection à un musée

en assortissant leur donation de conditions

strictes comparables, en particulier quant à la

présentation des objets. Ceci parce que, dans

les deux cas, ils considéraient leurs collections

comme des outils de démonstration de leurs

théories scientifiques. À partir de l'analyse

des formes des objets de sa collection

ethnographique, Pitt-Rivers a proposé

une véritable philosophie du progrès,

les formes et les opérations mentales dont

elles résultent obéissant aux mêmes lois de

l'évolution que celles des organismes vivants.

Géologue puis préhistorien, Piette avait

pour ambition majeure d'établir une synthèse

chronologique de la préhistoire à partir de

la juxtaposition des assemblages industriels.

Cet article examine ces théories, la façon

dont leur mise en scène muséographique

fut conçue et ce qu'il en reste aujourd'hui. 
1. Voir le site « Rethinking Pitt-Rivers » : http://web.prm ox.ac.uk/rpr

\section{ci-contre}

fig. 1

La salle Piette au Musée d'Archéologie nationale,

v. 1957. (C) archives MAN/

Charles Hurault.
Augustus Lane Fox Pitt-Rivers (1827-1900) a légué sa collection au musée d'Oxford qui porte aujourd'hui son nom. C'est un des plus importants musées d'ethnographie, avec quelque 300000 objets. Édouard Piette (1827-1906) a fait don de ses objets au musée d'Archéologie nationale de Saint-Germain-en-Laye, où ils sont conservés dans une salle du même nom; avec environ 8000 pièces, c'est un des plus prestigieux ensembles d'art mobilier préhistorique au monde (on y trouve notamment la célèbre Dame à la capuche de Brassempouy).

Piette et Pitt-Rivers, considérant leurs collections comme la démonstration de leurs théories scientifiques, assortirent tous deux leur donation d'un certain nombre de conditions strictes fort comparables, en particulier quant à l'agencement et la présentation des objets. II y a toujours des conceptions théoriques derrière la réalisation d'un musée, mais dans leur cas c'était explicite et la présentation même des objets participait de la démonstration. Tous deux ont mis en scène leurs conceptions de l'évolution de l'histoire de l'homme à travers leur musée. En partant de séries d'objets pour la plupart techniques - principalement d'origine ethnographique pour Pitt-Rivers, archéologique pour Piette -, ils ont développé des théories différentes. Piette avait pour ambition majeure d'élaborer une chronologie détaillée de la préhistoire, c'est-à-dire de mettre au point un calendrier de ses grandes étapes. Pitt-Rivers cherchait à reconstituer l'avancée intellectuelle et technique de l'homme à partir des formes des objets qu'il avait créés. II n'avait pas le souci de la chronologie développé par Piette et utilisait aussi bien des objets de provenance archéologique qu'ethnographique pour illustrer ses séries évolutives.

Aujourd'hui, tant le Pitt-Rivers Museum que la salle Piette sont considérés comme des musées dans le musée, dans le plus pur style victorien pour le premier, dans le style Napoléon III pour le second. Si l'apparence générale des salles d'exposition est bien d'époque, la disposition des objets dans les vitrines est loin d'être conforme à ce qu'avaient voulu les donateurs et nous verrons que l'un et l'autre ont été très vite trahis par leurs successeurs. Après une présentation de leurs théories et de leurs projets muséographiques respectifs, je tenterai de mettre en évidence ce qu'il reste aujourd'hui de la présentation des objets telle qu'ils l'avaient voulue. Malgré les nombreuses modifications réalisées depuis plus d'un siècle, on peut en effet avoir quelque idée de ce qu'ils avaient en tête et de l'aspect originel des vitrines. Je rappellerai les grandes lignes de I'histoire du Pitt-Rivers Museum reconstituée par l'équipe de chercheurs du musée ${ }^{1}$. Celle de la salle Piette est plus chaotique et je tenterai d'en retracer les grandes étapes et les principaux changements qui y ont été opérés.

\section{Des parcours et des théories distinctes}

Les deux hommes sont nés la même année, en 1827, Édouard Piette dans les Ardennes, Augustus Lane Fox Pitt-Rivers dans le Yorkshire. Piette est décédé en 1906, Pitt-Rivers en 1900. Le premier a été géologue avant d'être archéologue, tandis que le second a été archéologue parallèlement à ses activités de collectionneur. Comme c'était alors l'usage, tous deux exerçaient une activité professionnelle par ailleurs: Piette était juge de paix, Pitt-Rivers militaire. 





2. C'est précisément le titre des trois volumes de Jacques Boucher de Perthes, Antiquités celtiques et antédiluviennes, parus en 1847, 1857 et 1864 . Ces périodes annonçaient la division entre Paléolithique et Néolithique introduite par John Lubbock dans son ouvrage Prehistoric Times, paru en 1865 et deux ans plus tard en français.

\section{Édouard Piette}

Après des études de droit à Paris, Piette exerça comme juge de paix dans sa région d'origine de 1860 à 1879. Avant de se consacrer à la préhistoire, il s'est passionné pour la géologie et a publié, dès 1855, plusieurs articles sur le Jurassique du nord-est de la France. Très tôt intéressé par l'archéologie, il fouilla des nécropoles gauloises et mérovingiennes dans la région de Craonne, dans l'Aisne, mais la guerre de 1870 vint interrompre ses recherches. Le tournant dans sa carrière scientifique s'est produit en 1871 , quand des ennuis de santé l'obligèrent à faire une cure à Bagnèresde-Luchon. II s'intéressa tout de suite à la géologie des Pyrénées et s'interrogea sur l'existence de l'homme au moment du retrait des glaciers. II entreprit alors des fouilles intensives de 1871 à 1875 dans plusieurs grottes, dont Gourdan (Haute-Garonne), Lortet (Hautes-Pyrénées) et Espalungue à Arudy (Pyrénées-Atlantiques) [Delporte 1987: 18]. En 1879, toujours juge de paix à Craonne, il fut muté à Eauze, dans le Gers. II mit à profit sa nouvelle affectation pour s'intéresser au passé gallo-romain de la ville. De 1883 à 1891, date à laquelle il prit sa retraite, il semble avoir poursuivi des recherches sur le terrain dans les Pyrénées et réalisa en particulier, de 1887 à 1897, les deux plus importantes fouilles de sa carrière: celles des grottes du Mas d'Azil et de Brassempouy.

Parmi tous les sujets qui l'ont intéressé, signalons la question du «hiatus» entre les âges de la Pierre taillée et de la Pierre polie qu'il est le premier à avoir résolue en montrant qu'il a bien existé des assises de transition entre les deux époques. Cette question, controversée pendant toute la seconde moitié $d u x x^{e}$ siècle, résultait du fait que les niveaux séparant ce qu'on appelle aujourd'hui le Paléolithique et le Néolithique semblaient stériles. On en avait déduit que l'Europe occidentale avait été abandonnée pendant plusieurs millénaires. Cet abandon se manifestait par une discontinuité, un hiatus dans l'évolution des hommes et des techniques. Cela correspondait à la vieille idée émise par Jacques Boucher de Perthes dès $1847^{2}$ de l'existence de deux périodes - celtique et antédiluvienne -, dont les vestiges étaient séparés par des couches appelées «diluvium rouge» ou par d'épais planchers stalagmitiques qui témoignaient d'un cataclysme dans lequel certains continuaient à voir le déluge biblique. Les fouilles de Piette dans les grottes du Mas d'Azil et de Gourdan en 1887 et 1888 mirent fin à la controverse.

À partir des années 1880 et jusqu'à la veille de sa mort, Piette continua à publier de nombreux articles et travailla à un grand projet d'ouvrage sur L'Art pendant l'âge du Renne destiné à présenter les grandes phases de l'art paléolithique mobilier. Les planches, achevées en 1892, furent présentées avec sa collection en 1900 à l'Exposition universelle de Paris, mais l'ouvrage ne parut qu'en 1907, quelques mois après sa mort. L'ambition majeure de Piette a été «beaucoup moins de réunir une collection que de faire une étude détaillée des couches et de lire dans leur superposition la succession des temps, les progrès de l'industrie et la marche des sociétés humaines » (Piette 1895: 239). II partait de l'idée qu'à un groupe humain particulier correspondait un bagage technique donné. Les sociétés n'évoluaient donc pas mais se succédaient dans le temps. En bon géologue, il considérait qu'il suffisait de ranger les assemblages archéologiques selon leur ordre 
de dépôt stratigraphique pour comprendre la succession des populations et le progrès de l'humanité. Il utilisa, pour ce faire, les très nombreux objets préhistoriques qu'il avait découverts lors de fouilles étonnamment fructueuses. Sa collection comprend plus de 300 œuvres figuratives et au moins autant d'œuvres à décor géométrique. Elle comporte en outre de très grandes séries d'outils en pierre taillée et en matière dure animale, pour les seules collections paléolithiques (Schwab 2008: 12).

Rappelons qu'en cette seconde moitié du XIXe siècle, l'ancienneté de l'homme et sa contemporanéité avec des espèces animales disparues étaient admises depuis les années 1860, mais qu'une autre question se posait avec acuité, celle de la chronologie de ces temps préhistoriques: il s'agissait de préciser les grandes divisions de cette histoire ancienne de l'humanité et d'en établir une sorte de calendrier, ce qui devint la véritable obsession de tous les préhistoriens. En l'absence de datations absolues - qui ne seront permises qu'avec l'invention de la méthode de datation au radiocarbone en 1950 -, la stratigraphie constituait l'unique moyen d'ordonner les informations archéologiques sur une échelle chronologique. Les différents systèmes proposés suivirent l'exemple de la géologie, qui distinguait les époques par la présence de «fossiles directeurs", terme désignant les faunes fossiles servant de repères.

Sachant qu'au cours de cette longue période, des variations climatiques avaient entraîné des modifications dans le monde vivant, végétal et surtout animal, plusieurs voies apparaissaient possibles. Édouard Lartet (1801-1871) proposa de distinguer les époques par la prépondérance de certaines espèces animales ${ }^{3}$. Dès 1861, Piette proposa lui aussi un système chronologique fondé sur l'évolution de la faune. Il distinguait quatre âges - l'âge du Grand Ours des Cavernes, suivi de l'âge de l'Éléphant et du Rhinocéros, puis de l'âge du Renne, et enfin l'âge de l'Aurochs. Observant que ces différentes époques étaient également marquées par la présence de certains types d'outils, surtout en silex, Gabriel de Mortillet (1821-1898) préconisa, dès 1870, une chronologie fondée sur l'outillage, et dont les subdivisions portaient des noms rappelant la terminologie géologique, mais créés à partir de sites éponymes: Chelléen, Acheuléen, Moustérien, Aurignacien, Solutréen et Magdalénien.

À partir de 1889, Piette tenta d'améliorer la chronologie de la préhistoire en ajoutant aux séquences de Lartet et de Mortillet un troisième paramètre surtout utile pour la partie terminale du Paléolithique (à partir de -35000 à -30000 ans): les œuvres d'art mobilier ${ }^{4}$. L'originalité de sa classification vient du fait qu'il utilisait plusieurs critères à la fois là où ses prédécesseurs n'en avaient sélectionné qu'un seul. Le tableau de Piette fait ainsi état de la succession des climats, de l'apparition des beaux-arts, de l'avancement des techniques et des gisements éponymes.

Aussi ingénieux soit-il, son système chronologique n'a jamais été adopté par la communauté scientifique. D'abord parce qu'il l'a constamment modifié sous prétexte de l'améliorer et qu'il était donc impossible de suivre un système aussi mouvant. Piette publiait beaucoup, presque trop - une notule ou un court article à chaque nouvelle idée -, et sa pensée
3. Aujourd'hui perfectionnée et appelée biochronologie, cette méthode reste utile Iorsqu'une datation absolue est impossible. Elle consiste à découper le temps en biozones en tenant compte de la durée de vie limitée des espèces.

4. L'ancienneté de l'art pariétal n'a été reconnue qu'en 1901, ce qui explique que Piette n'en ait pas tenu compte dans sa chronologie. 


\section{GLASSIFICATION \& TERMINOLOGIE DES TEMPS PRÉHISTORIQUES \\ Par Édouard PIETTE}

\begin{tabular}{|c|c|c|c|c|c|}
\hline \multirow{2}{*}{$\begin{array}{l}\text { ĖRE } \\
\text { GROUPE } \\
\end{array}$} & \multirow{2}{*}{$\begin{array}{l}\text { TEMPS } \\
\text { SOUS- GROUPE }\end{array}$} & \multirow{2}{*}{$\begin{array}{l}\text { PÉRIODE } \\
\text { srstéme }\end{array}$} & \multirow{2}{*}{$\begin{array}{c}\text { AGE } \\
\text { sÉlite - sECTION }\end{array}$} & \multicolumn{2}{|c|}{$\begin{array}{l}\text { ÉPOQUE } \\
\text { ÉTAGE }\end{array}$} \\
\hline & & & & $\begin{array}{l}\text { Dénomination tirće đl'un fait } \\
\text { général caractéristique. }\end{array}$ & $\begin{array}{l}\text { Dénomination tirée du nom } \\
\text { d'une localité où } \\
\text { létage est bien téreloppé. }\end{array}$ \\
\hline \multirow{11}{*}{$\stackrel{[1}{\pi}$} & \multirow{6}{*}{ 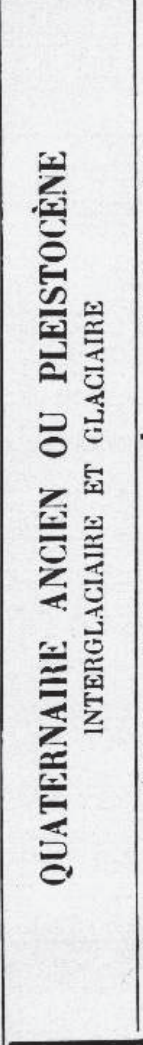 } & \multirow{2}{*}{ 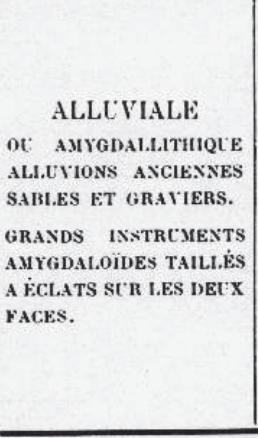 } & 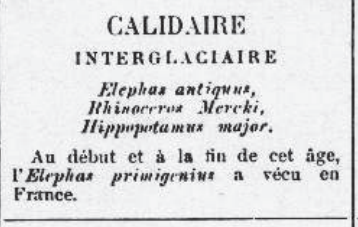 & $\begin{array}{l}\text { DE LA PREDOMINANCE DE } \\
\text { LELEPHAS ANTIQUUS }\end{array}$ & CHELLESIENNE \\
\hline & & & $\begin{array}{c}\text { FRIGIDAIRE } \\
\text { CHETES DE NEIGE } \\
\text { EXTENSIONS ET GRANDES OSCII- } \\
\text { L.ATIONS GLACIAIRES } \\
\text { Biephas primigerius. } \\
\text { Rhinacerus tichoriuns, } \\
\text { Hippipotawns amphibiun. } \\
\end{array}$ & $\begin{array}{l}\text { DE REFROIDISSEMENT } \\
\text { NON ENCORE } \\
\text { TRES INTENSE }\end{array}$ & ACHĖOLIENNE \\
\hline & & \multirow{4}{*}{ 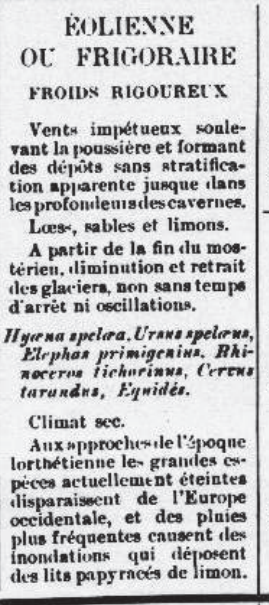 } & 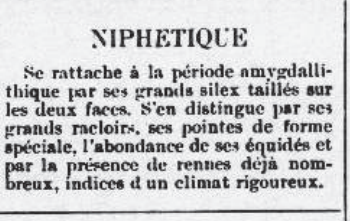 & $\begin{array}{l}\text { DE IA DERNIĖRE } \\
\text { GRANDE } \\
\text { EXTENSION GLACIAIRE } \\
\text { On ne trouve pas ses sidiments } \\
\text { sor le trajet des anciens glaciers } \\
\text { pyrénéens. }\end{array}$ & MOSTÉRIENNE \\
\hline & & & \multirow{3}{*}{ 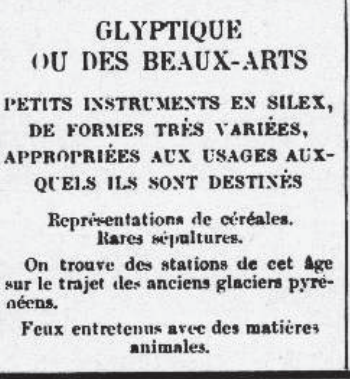 } & DE LA SCULPTURE & PAPALIENNE \\
\hline & & & & $\begin{array}{l}\text { DE LA GRAVURE } \\
\text { AVEC VESTIGBs } \\
\text { D'EsPĖCEs ÉTEIXTES }\end{array}$ & GOURDANIENNE \\
\hline & & & & $\begin{array}{l}\text { DE LA GRAVURE } \\
\text { SANS VEBTIGE D'ESPĖCES ETRINTES } \\
\text { XOMBREUX HARPONS } \\
\text { EN MOIS DE KENXE }\end{array}$ & LORTHÉTIENNE \\
\hline & \multirow{5}{*}{ 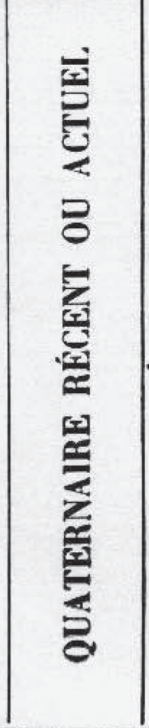 } & 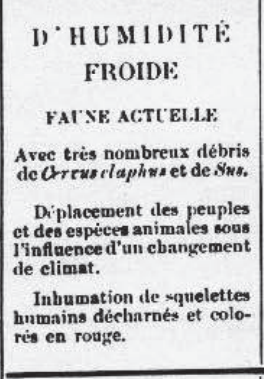 & $\begin{array}{c}\text { MÉTABATIQUE } \\
\text { OU DE TRANSITION } \\
\text { Persistance de nombreux instru- } \\
\text { ments en silex ile l'age glyptique. } \\
\text { Abondance vles harpons perforis, } \\
\text { plats, en ramure de cerf elapbe. } \\
\text { Petits tas de ble. } \\
\text { Inondations. Lits papyracés } \\
\text { de limon. } \\
\text { Feux entretenus avec des matieres } \\
\text { animales. }\end{array}$ & $\begin{array}{c}\text { DES GALETS C.OLORIES } \\
\text { EXTENSION DE LA VḱaḱtATION } \\
\text { AREOREBCETE } \\
\text { A LA FIN DE CETTE ÉPOQUB }\end{array}$ & ASYLIENNE \\
\hline & & \multirow{4}{*}{$\begin{array}{l}\text { DE CLIMAT } \\
\text { TEMPÉRE } \\
\text { FAUNE ACTUELIE }\end{array}$} & \multirow{2}{*}{$\begin{array}{l}\text { NÉOLITHIQUE } \\
\text { OU DE I.A PIERRE poI.IE } \\
\text { Immigration des peuples néolithiques } \\
\text { dnas la Ginvle. } \\
\text { Yeux de bois. }\end{array}$} & 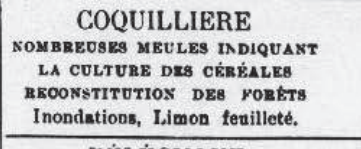 & ARISIENNE \\
\hline & & & & $\begin{array}{c}\text { PÉLECCYQUE } \\
\text { OU DES HACHES EN PIERRE } \\
\text { POLIE } \\
\end{array}$ & ROBENHAUSIRNNE \\
\hline & & & CALCEUTIQUE. DU BRONZE & & \\
\hline & & & $\begin{array}{l}\text { PROTOSIDERIQUE } \\
\text { PREMTER AGE DT ERR } \\
\end{array}$ & & \\
\hline
\end{tabular}


est ainsi apparue aussi complexe que confuse ${ }^{\mathbf{5}}$. Ainsi, le «Vallinfernalien» (nom créé à partir du site de Gorge d'Enfer) se trouvait dans la période qu'il appela éburnéenne, puis éléphantienne ou papalienne, correspondant à la phase initiale de son Glyptique ou Magdalénien (Delporte 1987: 151). Ensuite, la plupart des termes qu'il a créés étaient si insolites qu'il était impossible de les mémoriser. On est même allé jusqu'à le soupçonner d'une volonté d'hermétisme (ibid.: 157). Le tableau qui ouvre son ouvrage posthume (Piette 1907) donne une idée de cette nomenclature (fig. 2). Henri Delporte, conservateur au musée des Antiquités nationales dans les années 1980, s'est attelé à retracer l'élaboration de la pensée de Piette à partir de ses nombreuses notes et études mais il n'a pas pour autant réussi à réhabiliter ce chercheur déjà controversé à son époque (Delporte 1987).

Piette partageait l'opinion largement admise dans la seconde moitié $\mathrm{du} \mathrm{XIX}^{\mathrm{e}}$ siècle, selon laquelle les primitifs que l'on découvrait étaient les descendants directs des hommes de la préhistoire. Mais s'il les considérait comme d'un niveau technique équivalent, il avait davantage de considération pour les premiers hommes en qui il voyait de véritables pionniers:

[...] bientôt je fus convaincu que l'homme des cavernes, loin d'être un sauvage, avait été un pionnier de l'humanité, un être progressif qui avait posé les premiers fondements de notre civilisation. La caractéristique des sauvages est l'immobilité; ils reçoivent leur outillage de leurs pères; ils le transmettent à leurs descendants sans modification. Tels ne furent pas les hommes des temps glyptiques. (Piette 1907: 53)

Cette idée est pourtant en contradiction avec celle de populations qui se seraient succédé et remplacées sans montrer d'évolution interne.

\section{Le général Augustus Henry Lane Fox Pitt-Rivers ${ }^{6}$}

À la fois militaire et homme de science, le général Augustus Henry Lane Fox Pitt-Rivers participa aux missions des armées de sa Majesté britannique et prit part en particulier à l'expédition de Crimée en 1850 en qualité d'officier d'état-major. Sa spécialité était la balistique, et sa tâche sur le terrain était d'instruire les officiers sur l'usage des armes à feu (Thompson 1977; Bowden 1991). Le général était aussi bien collectionneur qu'archéologue. II comprit très tôt que la position relative des artefacts les uns par rapport aux autres pouvait être liée à la chronologie des niveaux successifs d'occupation et contribua au développement de la méthode stratigraphique (Bowden 1991), mais il ne l'a pas utilisée dans ses travaux relatifs à la technologie ${ }^{7}$.

La collection de Pitt-Rivers a constitué l'instrument principal de la méthode fondée sur l'analyse des formes qu'il a préconisée. À partir des années 1850 et durant une vingtaine d'années, il a rassemblé une gigantesque collection d'objets en tout genre, provenant des sociétés «sauvages" mais aussi de sites archéologiques. La collection dont il a fait don au musée d'Oxford comprenait quelque 20000 objets, dont environ 15000 provenaient du South Kensington Museum à Londres, section de Bethnal Green ${ }^{8}$. Après la donation à Oxford, il a continué à collecter des objets de 1880 à 1900 pour son nouveau musée installé à Farnham, dans le Dorset. On évalue à environ 50000 objets le total de ses deux collections.
5. Pour une bibliographie exhaustive d'Édouard Piette, voir Delporte 1987 : 165-174.

6. En France, c'est François Sigaut qui a sorti Pitt-Rivers de l'oubli, lors d'un séminaire de l'École des hautes études en sciences sociales consacré à la naissance et au développement de la technologie. Il en a tiré un article paru dans Gradhiva en 1990. William R. Chapman s'est intéressé à l'aspect muséographique de l'œuvre (1981 et 1991) tandis que Mark Bowden, auteur d'une excellente biographie, s'est attaché à l'apport de Pitt-Rivers dans le domaine de l'archéologie (1991). Par ailleurs, l'équipe du Pitt-Rivers Museum publie de nombreux travaux sur son œuvre et sa collection (site «Rethinking Pitt-Rivers »: http://web.prm.ox.ac.uk/rpr/). Récemment, un article de Carlo Severi (2003), consacré plus généralement à ce que l'auteur appelle la biologie des images, lui fait une large place et un autre, de Nathan Schlanger (2010), s'insère dans un numéro de revue sur la sérialité.

\section{Soulignons que} Pitt-Rivers n'utilise pas le terme «techniques" mais son équivalent à l'époque, material arts.

8. Nom du premier musée où étaient exposés ces objets avant d'être donnés au Kensington Museum.

\section{ci-contre}

fig. 2

Classification et terminologie des temps préhistoriques d'Édouard Piette, état de 1906, (Piette 1907). 
9. Il signe ses premiers articles Lane Fox puis change son nom en 1880 pour adopter celui de Pitt-Rivers, «selon le désir du second Lord Rivers " (Pitt-Rivers 1891, n. 1), après avoir pris possession de l'héritage familial - un immense domaine dans le Dorset. Son activité archéologique, débutée aux débuts des années 1860, se poursuit alors à partir de fouilles conduites sur ses propres terres.
Pitt-Rivers a multiplié les conférences et les allocutions diverses pour promouvoir son système de classification et sa théorie sur l'évolution des objets techniques. L'essentiel de sa pensée, d'une cohérence et d'une constance remarquables, est exposé dans les trois conférences qu'il a données en 1874-1875, republiées dans le seul volume paru sous son nom, mais à titre posthume, avec trois autres textes (Pitt-Rivers 1906). II considérait que la présentation de sa collection suffisait à démontrer la validité de sa théorie des objets et il y voyait, peut-être plus que dans ses écrits, l'expression la plus achevée de ses recherches (Sigaut 1990: 35). Si sa théorie n'est pas passée à la postérité, c'est sans doute parce que très tôt l'organisation de son musée n'y a plus été conforme. Comme il avait peu publié, il ne resta que peu de traces de ses idées.

D'après lui, le développement de la science - l'organized common sense - passe par trois phases successives: un empirical or practical stage correspondant au sens commun inorganisé dans lequel nos connaissances résultent de l'expérience ordinaire, un classificatory or comparative stage, où les sujets étudiés sont regroupés en classes selon certaines affinités afin de pouvoir être comparés, et un theoretical or evolutionary stage (Lane Fox ${ }^{9}$ 1875b: 357-359) que Sigaut propose de traduire par «génétique » car il s'agit de s'y interroger sur la façon dont sont engendrés les phénomènes (Sigaut 1990: 31). Nous intéressent ici les stades classificatoire et évolutionnaire.

Dans une conférence donnée en 1874, Pitt-Rivers présente la classification de sa collection en quatre grandes catégories d'objets (Lane Fox 1875a: 293). La première consiste en une petite collection de crânes et cheveux dont les traits sont supposés permettre de distinguer les différentes «races » humaines. N'étant pas expert en la matière, Pitt-Rivers n'en dit pas plus et précise que les anthropologues ont largement traité de cette question. Sa deuxième catégorie réunit un grand nombre d'armes des existing savages. La troisième regroupe l'ensemble des techniques des sauvages modernes, de la poterie aux modes de navigation et aux textiles en passant par la parure, l'art, les outils, les ustensiles et les mobiliers domestiques, les emblèmes religieux, les monnaies, etc., et même les armes à feu. La quatrième, inattendue, réunit des objets préhistoriques - outillage paléolithique et néolithique, en bronze, fer et os - et un certain nombre de formes naturelles simulant des formes artificielles, ainsi que des contrefaçons modernes. Ceci parce que Pitt-Rivers pensait que les plus anciens outils préhistoriques étaient dérivés de formes naturelles, d'abord utilisées telles quelles par l'homme avant qu'il sache les reproduire. On sait bien aujourd'hui que bon nombre d'outils, qu'il s'agisse des outils tranchants ou encore de la roue tournant sur son axe, n'existent pas dans la nature (Sigaut 1990). Pitt-Rivers commettait ici une erreur, que l'on pourrait qualifier d'illusion rétrospective, en pensant que l'homme pouvait reconnaître dans des objets naturels la forme d'objets artificiels qui n'existaient pas encore.

Pitt-Rivers a tenté de ranger les objets des deux dernières catégories en séquences évolutives - c'est son evolutionary stage - afin de retracer l'histoire naturelle de la pensée humaine, ou plutôt sa préhistoire, à des époques dont l'écriture ne pouvait témoigner. Dans ce même texte (Lane Fox 1875a), il formule très clairement l'essentiel de son programme 
de recherche, qui consiste à transposer les idées de Charles Darwin sur l'évolution des organismes vivants aux productions de l'esprit humain afin de reconstituer la séquence des idées qui a permis à l'humanité de passer de la condition des animaux inférieurs à celle d'aujourd'hui, et d'établir ainsi des matériaux vraiment solides pour une philosophie du progrès (ibid.: 300 ).

Cette théorie du progrès repose sur l'intuition que les formes des objets reflètent des idées. II fait ainsi l'hypothèse: 1 , qu'il est possible d'établir des liens entre différents objets à partir d'une analyse de leur forme; 2 , que ces relations entre formes sont cruciales parce qu'elles permettent de reconstruire les opérations mentales qu'elles impliquent; 3 , que les idées s'engendrent les unes les autres, si bien qu'elles obéissent aux mêmes lois que celles de l'évolution des êtres vivants. C'est pourquoi il sélectionne les objets ordinaires et typiques plutôt que les spécimens rares, pour les arranger en séquences afin d'illustrer la succession des idées ayant présidé à leur élaboration. Comme les produits du règne animal et végétal, les idées humaines peuvent être classées en genres, espèces et variétés, et leur évolution retracée, du simple au complexe et de l'homogène à l'hétérogène, puisqu'elles obéissent à la même loi (ibid.: 307). Pitt-Rivers va plus loin en avançant que les idées nouvelles sont produites par la corrélation d'idées préexistantes, de même que les individus nouveaux d'une lignée résultent de l'union d'individus préexistants. Et de conclure: «Le progrès est comme un jeu de dominos - le semblable s'adapte au semblable. » (Ibid.: 308)

En vertu du principe de continuité qui fait que les idées s'engendrent les unes les autres, l'homme n'inventerait que très rarement et ne ferait qu'adapter. Chaque groupe humain aurait ainsi reçu de l'extérieur l'essentiel de ce qui fait sa civilisation. Pitt-Rivers en déduit que, plus on remonte dans le temps, plus les hasards favorables à l'invention ont dû être rares, et donc plus le rythme du développement de la civilisation a dû être lent. Il en conclut logiquement à la très haute antiquité de l'homme (Sigaut 1990: 27). N'oublions pas que nous sommes à une époque où l'idée d'une intervention divine dans l'apparition et l'histoire de l'homme faisait encore débat.

Pour expliquer la façon dont les idées se seraient engendrées, PittRivers s'inspire des Principes de psychologie d'Herbert Spencer (1855). Comme lui, il distingue, dans l'activité de l'esprit humain, les capacités conscientes et intentionnelles de celles qui nous permettent d'agir sans que ni la volonté ni la conscience ne soient convoquées. L'activité mentale automatique - que Pitt-Rivers appelle automaton mind - fonctionne de manière instinctive et inconsciente, et résulte de la réitération d'une habitude: il en est ainsi de la marche, qui est pourtant le fruit d'un dur apprentissage dans l'enfance. De même, si l'écriture et la lecture finissent par devenir automatiques, leur acquisition requiert un patient et difficile effort intellectuel. Selon Spencer, une fois qu'il est maîtrisé, un mode d'action conscient et réfléchi est transféré au niveau instinctuel de l'esprit. Pitt-Rivers transpose l'expérience individuelle à celle des sociétés et fait l'hypothèse qu'il en est de même pour les actions devenues automatiques chez les "sauvages" modernes, alors que ce sont leurs ancêtres préhistoriques qui ont fourni un effort intellectuel pour les inventer (Lane Fox 1875a: 296). Ainsi, comme Piette, il estimait que si les «sauvages» contemporains sont les descendants 
10. À l'exception de quelques vestiges provenant de ses propres fouilles, très minoritaires par rapport aux objets archéologiques et ethnographiques qu'il achetait. directs des populations préhistoriques, ils n'en ont plus les capacités inventives. En outre, puisque les formes et les opérations mentales qui les régissent obéissent aux mêmes lois de l'évolution que celles des organismes vivants, les idées des humains ont, comme les espèces animales, une distribution géographique et chronologique particulière. Ainsi, les formes évoluent non seulement par leur perfectionnement dans un groupe donné, mais aussi lors du transfert d'une population à une autre ou d'un lieu à un autre. L'observation de l'évolution des formes présente donc un grand intérêt sociologique pour comprendre les liens entre populations (ibid.: 295).

Dans un récent article, Nathan Schlanger (2010) avance l'hypothèse que Pitt-Rivers s'est inspiré des méthodes de classification de la numismatique, et en particulier des travaux du numismate, antiquaire et géologue John Evans. Evans se fit connaître en 1850 par un article dans lequel il prétendait que les monnaies britanniques indigènes avaient précédé les monnaies romaines, leur prototype étant la statère de Philippe de Macédoine ou, plus probablement, son imitation gauloise. En plaçant sur une planche une sélection de pièces, Evans montrait comment, à partir de ce prototype, et par le moyen d'imitations successives, plusieurs types nouveaux et totalement distincts avaient émergé, jusqu'à ce que leur origine soit perdue. Pitt-Rivers cite effectivement Evans à plusieurs reprises et reproduit même une de ses planches - avec la permission de l'auteur - afin d'illustrer sa théorie de l'évolution progressive des formes (Evans 1875, in Lane Fox 1875a), alors que sa démonstration était pourtant déjà éclairée par ses propres schémas.

Quoi que Pitt-Rivers ait emprunté à Evans dans l'élaboration de ses idées sur l'évolution des techniques, il se pourrait bien cependant que ses intuitions premières soient nées d'une expérience personnelle, qu'il a relatée dans un article de 1891. Quarante ans plus tôt, en 1852, étant encore un jeune sous-officier, il avait été chargé de rédiger les notices de nouvelles armes soumises à un comité de sélection. II devait noter les légers changements qui caractérisaient les différentes inventions et améliorations proposées. C'est à ce moment que lui vint l'idée de monter une collection d'armes présentant tous les stades successifs d'amélioration dans leur ordre d'apparition. Et c'est ainsi que débuta sa collection. II n'aurait convoqué la théorie de la dérivation des formes proposée par Evans qu'ensuite, pour donner plus de poids à sa propre argumentation.

On comprend mieux pourquoi Pitt-Rivers a fait une si large place aux armes dans sa réflexion sur la technique. Ainsi sa carrière militaire - il a été plutôt ingénieur d'armement et instructeur que combattant (Sigaut 1990: 22) - et sa passion de collectionneur se rejoignaient autour de son intérêt pour la balistique.

\section{Le musée comme administration de la preuve}

Nous avons vu que, si les deux hommes étaient collectionneurs, Piette trouvait ses objets dans les gisements qu'il fouillait (sauf très rares exceptions) alors que Pitt-Rivers les achetait ${ }^{10}$. La manière dont l'un et l'autre enrichissaient leurs collections est directement liée à l'usage qu'ils en faisaient: Piette ne choisissait pas ses objets et était tributaire de la richesse des sols archéologiques; Pitt-Rivers les achetait en fonction de ses besoins pour 
compléter ses séries évolutives, exerçant une sélection en amont, vraisemblablement selon un schéma théorique préalable d'une succession de formes qu'il avait en tête. Tous deux étaient néanmoins archéologues et on peut s'étonner que Pitt-Rivers, dont on sait qu'il était sensibilisé à l'importance de la succession des couches géologiques, n'en ait pas tenu compte pour classer les objets de sa collection. C'est qu'il n'en avait tout simplement pas besoin puisque seule lui importait l'évolution des formes, indépendamment de leur chronologie. Quant à l'intérêt formidable de Piette pour la succession chronologique des couches d'enfouissement des vestiges, il peut s'expliquer par sa formation de géologue, dont il tirait une grande fierté.

Pour comprendre les divergences de posture des deux savants, on peut aussi alléguer, en suivant Claudine Cohen, que les archéologues anglais de cette époque étaient très versés dans la spéculation et le comparatisme ethnographique, à l'instar de James Frazer, alors que les préhistoriens français étaient plus terre à terre, peut-être en raison de la richesse du sol français en vestiges préhistoriques (Cohen 2011: 231). Mais tous deux ne s'intéressaient à leur collection que dans la mesure où ils pouvaient en faire un outil démonstratif: “Ma collection n'est pour moi qu'un instrument de travail », disait Piette (lettre du 7 mai $1903^{11}$ ), qui voulait ordonner les objets pour reconstituer la succession des cultures humaines et «l'histoire de l'humanité à l'âge glyptique dans le pays de Gaule» (lettre du 20 novembre $1902^{\mathbf{1 2}}$ ). De même, Pitt-Rivers préconisait l'utilisation du musée pour illustrer l'évolution (Pitt-Rivers 1891) et rendre explicite une théorie élaborée des relations de causalité interne qui unissaient les objets et des opérations mentales présidant à leur élaboration. II était donc logique que tous deux attachent une grande importance à l'agencement des objets dans les vitrines. En plus d'administrer la preuve de la cohérence de leur théorie, ils considéraient que le musée avait aussi une vocation didactique, et c'est pourquoi ils tenaient à ce que leurs collections soient accessibles au public.

Les deux savants avaient fait de leur musée un véritable enjeu scien-tifique et c'est pourquoi leur donation fut assortie de contraintes très strictes. Sans entrer ici dans les détails juridiques, retenons qu'ils exigèrent une salle à part portant leur nom (dans le cas de Pitt-Rivers, ce sera un bâtiment séparé), l'accessibilité au public, la présentation exhaustive de la collection (à l'exclusion de toute autre dans le cas de Piette) et le respect de la disposition des pièces sans qu'aucune modification soit apportée dans la présentation ${ }^{13}$. Piette avait pour ambition d'établir une classification et une terminologie de la préhistoire qu'il considérait comme définitive et c'est pourquoi il souhaitait qu'aucun remaniement ne soit effectué après sa mort. Moins rigide, Pitt-Rivers demanda que tout changement soit fait avec son autorisation de son vivant, mais il était précisé qu'après sa mort seules les modifications faisant avancer le cours de la science devaient être envisagées. II se désintéressa en fait de sa collection dès la donation effectuée pour se consacrer à la "seconde collection » qu'il commença à réunir dans son nouveau musée du Dorset. Ces postures sont à mettre en lien avec la manière dont tous deux acquéraient leurs objets: une fois extraite du sol, une série d'objets archéologiques ne peut plus être modifiée alors qu'à l'inverse une collection qui s'enrichit «au fil de l'eau» peut à tout moment remettre en cause l'ordre de la série évolutive.
11. Archives du musée d'Archéologie nationale (MAN), fonds Piette.

12. Archives MAN, fonds Piette.

13. Pour les termes de l'acte de donation de Pitt-Rivers signé le 20 mai 1884, voir http://web.prm.ox.ac. uk/rpr, Primary Documents, Founding Collection 18501900. Pour Piette, voir l'acte de donation daté du 25 février 1904 (archives MAN, fonds Piette) et Schwab 2008. 
178

études et essais

3

me collection as musé de daint-Germain, masia' certaines conditions que je vair énumerer.

$1^{\circ}$. . demande une subvention pour solder les frais de tirage des plareches de mom album D. $P^{\prime}$

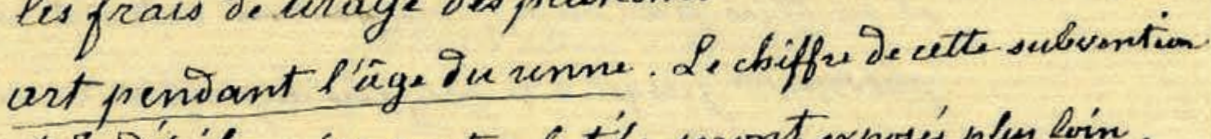

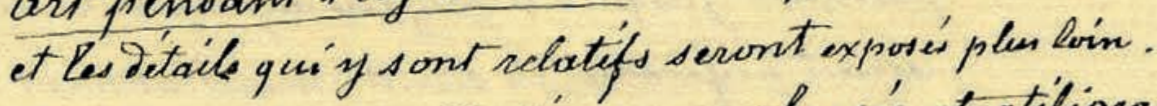

$2^{\circ}$ Ha collection sera classé stratifigraphiquemunt et par grottes, comme etle l'est thes moi, conformément à ma clussification dorat le nums $D$. périvdes, áges, éporecess, systeme, série, assise, étayes

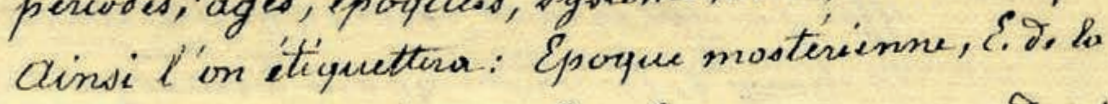

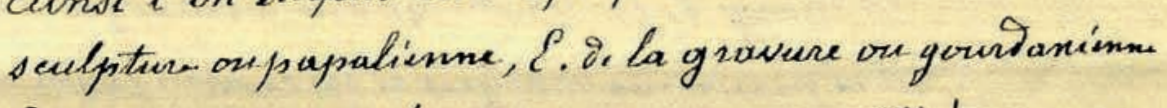

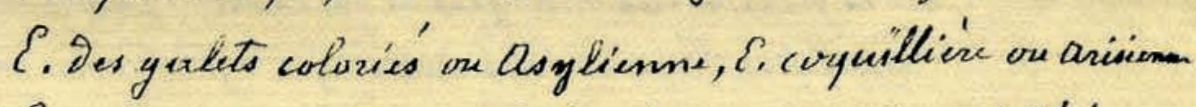

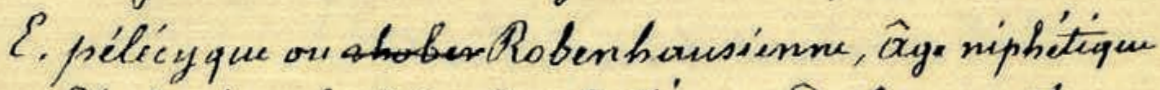

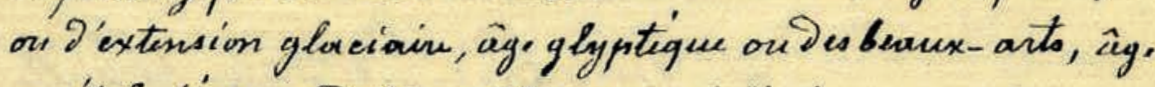
métabutique ouग, transition, ay-nédithique va $\partial$ la pierse polie, äge caleutiqu, ôg-protosisenique, Periog. calidaine,

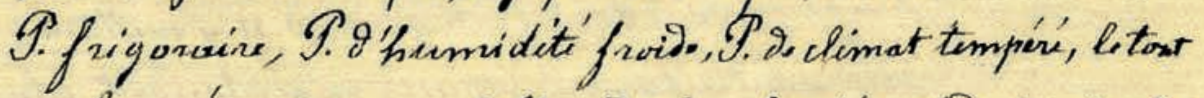
conform ément a mon tablian ir classificution ext. terminulgi:

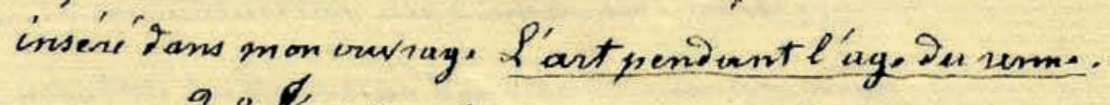

3 - Wutes les gnavures, sculptures, peintures Eoves fes instruments, critelo et armes en os, ivvire, bois?.

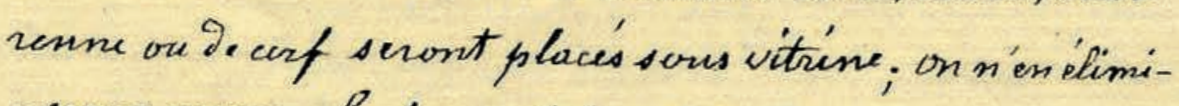
- nera erecun. La frume et les silex \%. Brassempersy sonent expores en enter sous vitrine. In ayer concerme ba faume 


\section{Mise en scène muséographique de la chronologie préhistorique voulue par Piette}

La correspondance de Piette, l'acte de donation et le catalogue de l'Exposition universelle de 1900 permettent de comprendre assez précisément la manière dont il souhaitait que sa collection soit présentée. Son exigence principale, sur laquelle il est maintes fois revenu, était que les objets soient présentés par couche (ou «assise») stratigraphique, en ne tenant compte du "gisement» dont ils provenaient que dans un second temps - de la même manière que les fossiles géologiques ne sont présentés dans un musée, aujourd'hui encore, que par rapport à la strate géologique dont ils ont été extraits, leur localité de découverte n'étant indiquée que secondairement et à l'intérieur d'un niveau donné. Cette façon de présenter les vestiges archéologiques était déjà considérée comme suffisamment étrange à l'époque pour que Piette ait eu le plus grand mal à la faire admettre. L'usage voulait en effet que les collections soient présentées par site et par type d'objet, comme c'est encore le cas aujourd'hui dans la plupart des musées. Et la salle Piette, restaurée en 2008 précisément pour être en totale conformité avec l'acte de donation, ne présente pas la collection de la manière voulue par Piette. Nous tenterons de comprendre pourquoi.

Piette a clairement exprimé ses exigences quant à la présentation de sa collection dès 1902, dans la lettre du 21 juin contenant son projet de donation:

Ma collection sera classée stratigraphiquement et par grottes, comme elle l'est chez moi, conformément à ma classification, avec les noms de périodes, âges, époques, système, série, assise, étages. [...] le tout conformément à mon tableau de classification et de terminologie inséré dans mon ouvrage L'Art pendant l'âge du Renne ${ }^{\mathbf{1 4}}$ (fig. 3).

II a ensuite réitéré cette exigence dans plusieurs autres courriers ${ }^{\mathbf{1 5}}$. Dans une lettre de novembre 1902, le mode de regroupement des objets par assise stratigraphique est très explicite:

[...] une seule étiquette indiquant l'assise est placée pour tout un groupe d'objets trouvés dans cette assise, groupe que l'on a soin de séparer des autres par des baguettes de bois. L'indication de la localité est seule indiquée sur des étiquettes placées près de chaque objet ${ }^{16}$.

Il est significatif qu'il utilise ici le terme «localité» (ou ailleurs celui de «gisement ») plutôt que celui de site, la localité ou le gisement, en géologie comme en zoologie et en botanique, étant le lieu où se trouve un spécimen donné. Pour lui, l'objet n'a d'intérêt qu'en tant que témoin d'une assise géologique.

Dans une autre lettre écrite trois jours plus tard ${ }^{\mathbf{1 7}}$, il insiste sur le fait qu'il faudra prendre exemple sur la façon dont ses collections ont été présentées lors de l'Exposition universelle du Trocadéro (1900) où, d'après le catalogue, les objets étaient disposés par assise stratigraphique et non par site (Capitan 1900: 297 sq.).

L'auteur du catalogue donne la liste des différents étages des vitrines et le détail de leur contenu: 1, étage de la sculpture (papalien) comprenant
14. Lettre du 21 juin 1902 adressée à Salomon Reinach, archives MAN, fonds Piette.

15. «Lors de l'exposition du trocadéro Mr d'Ault Dumesnil qui est géologue et qui avait pris des notes en emportant ma collection, l'avait exposée dans l'ordre stratigraphique. [...] La principale originalité de ma collection est d'être classée stratigraphiquement de manière que l'on puisse d'un seul coup d'œil se rendre compte de l'état des arts et de l'industrie, à une époque, et qu'en suivant l'ordre des vitrines, on puisse voir les progrès accomplis à cette époque par le perfectionnement des procédés et des instruments anciens et par l'invention d'instruments nouveaux. C'est en étudiant la stratigraphie des assises qu'on lit leur âge relatif et que l'on peut établir la chronologie préhistorique. Voilà pourquoi la classification doit être stratigraphique. [...]. Mais la collection devra rester classée stratigraphiquement parce que la chronologie est le fil conducteur de la science préhistorique. » (Lettre du 22 août 1902, archives MAN, fonds Piette)
16. Lettre du 26 novembre 1902 à Benoît Champion, chef des travaux du musée, archives MAN, fonds Piette.

17. Lettre du 29 novembre 1902, archives MAN fonds Piette.

\author{
ci-contre \\ fig. 3 \\ Lettre d'Édouard Piette \\ à Salomon Reinach du \\ 21 juin 1902, f. 3 C Musée \\ d'Archéologie nationale. \\ fonds Piette.
}


18. Acte de donation, 25 février 1904, archives MAN, fonds Piette.

19. Lettre du 21 avril 1901, archives MAN, fonds Piette. l'assise de la sculpture en ronde bosse et celle de la sculpture en bas relief; 2, étage de la gravure (gourdanien) se déclinant en assise de la gravure à contours découpés, de la gravure sans harpons et de la gravure avec harpons. Les noms de sites sont à peine mentionnés et ce n'est que grâce à la description de certaines pièces très célèbres qu'il est possible aujourd'hui de les identifier: il est clair que les objets sont mélangés sans tenir aucun compte des gisements dont ils proviennent.

La vitrine consacrée aux objets plus récents respecte elle aussi l'ordre chronologique par «étage», selon la terminologie géologique de l'époque: 3, étage des galets coloriés (ou asylien); 4, étage coquillier (arisien); 5, étage pélécyque (robenhausien); 6, période calceutique. La superposition se poursuit avec certains objets attribués à la période protosidérique (hallstattienne). Puis un niveau gaulois, une couche romaine et des dépôts du Moyen Âge: chaque couche est représentée par des séries de pièces "que nous avons choisies dans ses belles collections, de façon à ce que la démonstration fût aussi nette que possible» (ibid: 303). Cette vitrine se termine par une série de pièces provenant des fouilles de «MM. Piette et Laporterie» à Brassempouy. Louis Capitan précise bien qu'elles sont présentées à part, «et cette fois-ci, par site», traitement exceptionnel dû au fait qu'elles appartiennent «aux héritiers du propriétaire de la grotte, feu de Poudenx". Cette série regroupe des outils, des objets gravés et des statuettes de diverses périodes.

Pour mieux comprendre ce que Piette avait en tête, il faut revenir à l'acte de donation, daté de 1904, dans lequel il est bien précisé que les objets doivent être classés d'abord par assise stratigraphique, puis par catégorie d'objet ${ }^{\mathbf{1 8}}$. Les objets doivent être classés à la manière des fossiles, c'est-à-dire en tenant compte de l'étage géologique dont ils proviennent et non du lieu de leur découverte. Dans ce document, on apprend aussi que le niveau de classification suivant subdivise les vestiges par type d'objet en quatre catégories: beaux-arts, industrie de l'os, industrie du silex, faune. Piette n'envisage d'indiquer la provenance qu'ensuite, par le biais de petites étiquettes montées sur le support qui porte chaque pièce.

Par son classement stratigraphique, Piette s'oppose à ses pairs, qui privilégiaient un classement par site et par type. Sa formation initiale a sans doute conditionné ses habitudes de travail. En 1901, il écrit à l'abbé Breuil, alors un tout jeune homme: «Vous auriez très bien trouvé sans moi la méthode stratigraphique qui est la mienne, mais qui ne m'appartient pas en propre, puisqu'elle est celle de tous les géologues sachant leur métier ${ }^{19}$. »

\section{De la théorie à la mise en scène muséographique voulue par Pitt-Rivers}

Pour administrer la preuve de la validité de sa théorie de l'évolution des formes, Pitt-Rivers précise que les spécimens doivent être placés de manière à montrer comment une forme conduit à une autre. Comme nous ne disposons pas de date pour les most prehistoric objects, il faut avoir recours à des séquences par type, démarche qu'il propose d'appeler «typologie» (Pitt-Rivers 1891: 115). L'emploi de ce terme est alors controversé car l'usage du mot «type » a un sens bien précis en histoire naturelle (discussion 
in ibid 1891). II semble que ce soit Pitt-Rivers qui l'ait introduit en archéologie, ou qui du moins en ait fait admettre l'usage.

Puisque les objets sont les seuls témoins des opérations mentales qui ont présidé à leur production, leur disposition dans le musée montrera comment l'activité mentale des hommes a évolué au cours du temps. En partant des objets dus aux «sauvages» contemporains et en remontant jusqu'à ceux qui nous viennent des hommes préhistoriques les plus anciens, on peut retracer de proche en proche l'évolution de cette activité ${ }^{20}$. Il s'agit donc bien, comme Pitt-Rivers le dit lui-même, du principe de raisonnement scientifique consistant à aller du connu vers l'inconnu. Au lieu d'écrire l'histoire de l'humanité du passé vers le présent, en partant du simple pour aller vers le complexe, il tente de l'écrire à rebours. Carlo Severi (2003: 90) rapproche la démarche de Pitt-Rivers de la méthode de Zadig convoquée par Thomas Henry Huxley pour faire de la «prophétie rétrospective» (Cohen 2011: 20). Pour construire les lignées généalogiques des espèces, Huxley préconisait de remonter des formes actuelles connues aux ancêtres inconnus, c'est-à-dire des formes les plus évoluées aux plus primitives. Si la méthode de Zadig s'occupe avant tout de traces - l'empreinte de sabot pour retrouver le cheval, celle de la queue pour en inférer que le chien est de petite taille -, le parallèle est ici pertinent tant Huxley comme PittRivers étaient portés à voir dans les formes du présent les traces du passé. C'est sur l'évidence de cette évolution du préhistorique au «sauvage» contemporain que Pitt-Rivers fonde tout son système d'exposition: les objets doivent être disposés en lignées censées restituer de façon visible le développement mental et culturel de l'humanité.

Plus précisément, il préconise de répartir les objets à exposer selon de grandes classes correspondant à leur fonction ${ }^{21}$ : les arcs, les lances, les massues, les boomerangs, etc. Dans chaque classe, on les distinguera selon leur origine géographique, ce qui donnera autant de sous-classes dans lesquelles on regroupera les objets selon leur air de famille, en les alignant du plus simple au plus complexe (Lane Fox 1875a: 294-295) (fig. 4). Puisque les outils les plus primitifs étaient supposés dériver de formes naturelles, les premières armes avaient été de simples bâtons épousant les courbes naturelles des branches d'arbre. L'idée est illustrée par un schéma où Pitt-Rivers a présenté l'évolution des armes australiennes. La place de l'origine y est tenue par un bâton dont toutes sortes d'armes seraient issues: matraques de guerre, divers types de boomerangs et de massues, de boucliers et de bâtons de jet (fig. 5). Les spécimens sont bien répartis selon leur fonction, mais, dans ce cas d'espèce au moins, un outil unique est censé avoir donné naissance à des armes aux fonctions très différentes. Le bâton dont Pitt-Rivers fait la première arme humaine était déjà l'arme naturelle utilisée par les grands singes et les éléphants, avant même l'apparition de I'homme sur la Terre (ibid: 302).

À la fin de sa vie, Pitt-Rivers voulait généraliser ce système de présentation radiale, le mieux à même selon lui de rendre le caractère multilinéaire de l'évolution: le musée idéal serait une vaste anthropological rotunda (Pitt-Rivers 1888). II expose cette idée à l'Anthropological Section of the British Association à Bath en 1888, puis dans diverses publications
20. Pour les outils préhistoriques en matière périssable, Pitt-Rivers suggère de les remplacer en imagination en étudiant ceux de « his nearest congener, the modern savage " (Lane Fox 1875a: 295), ce qui est contradictoire si l'on admet avec lui qu'il y a évolution de l'outillage préhistorique à l'actuel.

21. Il parle non de fonction mais de "forme" (form) quoique, à l'évidence, ce soit bien de fonction qu'il s'agit. 

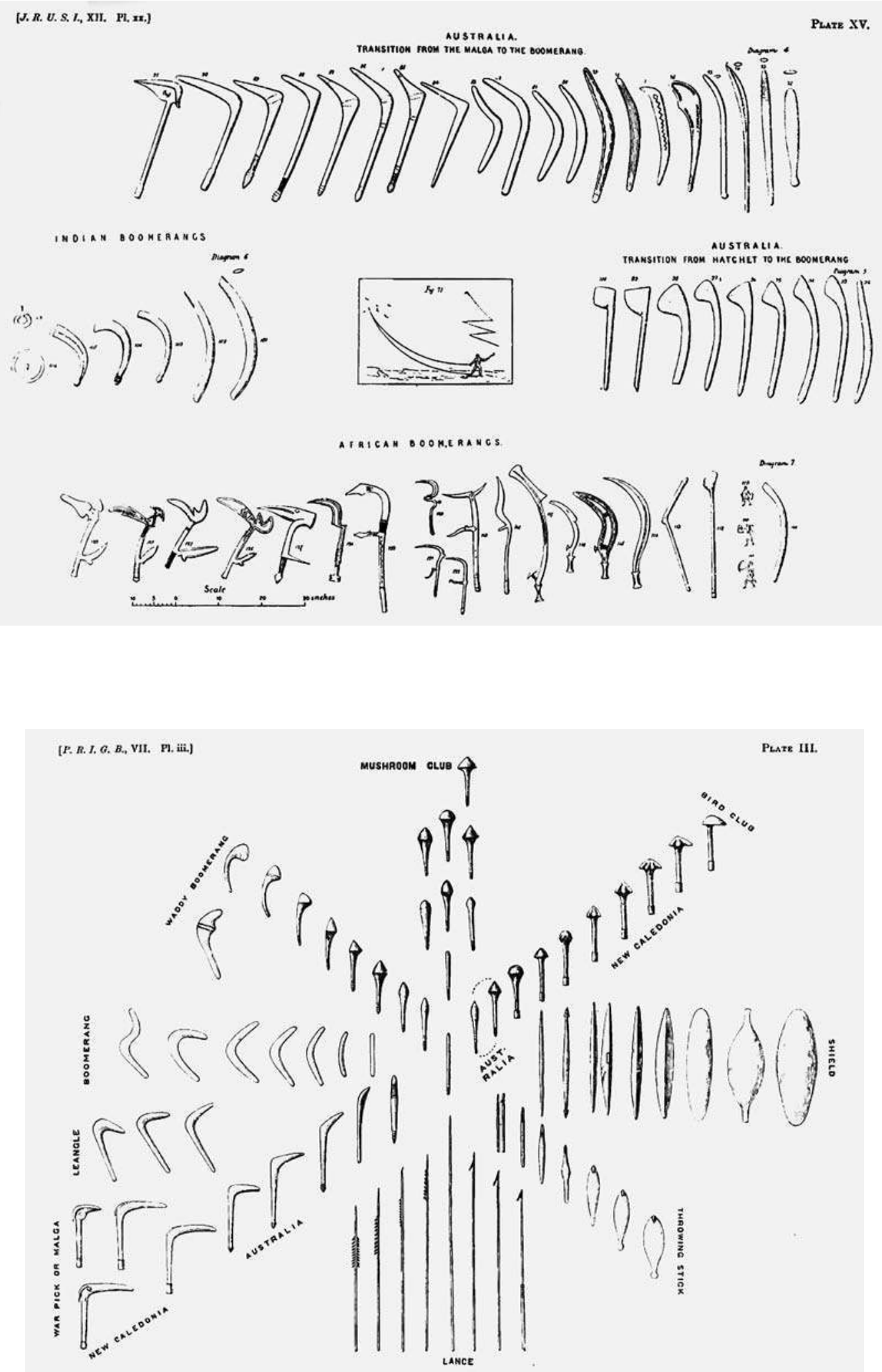
(1889-1890 et 1891). Les objets y seraient répartis en différents secteurs géographiques et disposés sur plusieurs cercles concentriques, un par période (Paléolithique, Néolithique, âge du Bronze, du Fer, etc.), dont le centre correspondrait à l'origine de l'humanité. Les sections et soussections seraient subdivisées par grandes catégories techniques: poterie, architecture, modes de navigation, matériel pour chevaux, outils, armes, matériel de tissage et textiles, métallurgie... L'étudiant le moins instruit pourrait ainsi comprendre facilement l'évolution de n'importe quel objet en se contentant d'observer sa distance par rapport au centre de la rotonde. Un tel musée nécessiterait de constants arrangements en fonction de l'évolution des connaissances. Pitt-Rivers signale que le professeur William Henry Flower, du Muséum d'histoire naturelle de Londres, a eu la même idée que lui pour présenter l'évolution des espèces naturelles et il en conclut que cette convergence de vue est le signe de l'évidence de cette idée (Pitt-Rivers 1891: 117). On voit ici que les deux objectifs du musée, scientifique et pédagogique, se rejoignent.

\section{Que reste-t-il aujourd'hui de l'argument scientifique voulu par les fondateurs de ces musées? Genèse et destin de la salle Piette}

Comme nous l'avons dit, la collection de Piette est conservée dans une salle spéciale du musée qui porte depuis le 27 juin 2005 le nom de musée d'Archéologie nationale, après s'être appelé Musée gallo-romain puis musée des Antiquités nationales. Créé en 1862 par la volonté de Napoléon III et installé dans le château de Saint-Germain-en-Laye, ancienne résidence des rois de France, il n'a été inauguré que le 12 mai 1880. La muséographie actuelle de la salle Piette est conforme à celle des musées de l'époque de Napoléon III (fig. 6). Exposés dans des vitrines en chêne très ouvragées, les objets sont disposés sur des fonds de feutrine rouge et maintenus par des griffes en laiton. Les petites pièces - aiguilles, pendeloques ou coquilles - sont placées dans des coupelles en verre. Pour répondre au souci d'exhaustivité prisé à l'époque, les objets sont très nombreux et serrés - parfois jusqu'à mille dans une seule vitrine. De fines baguettes de bois séparent les sections et les sous-sections. Des étiquettes manuscrites mentionnent le nom des sites, des périodes et des types d'objets (Schwab 2008).

Malgré cette apparente conformité à la pratique muséale du XIX siècle, les vitrines ont subi de nombreux changements depuis le moment où, dans des circonstances retracées en détail par Marthe Chollot (1964) puis par Delporte (1987), la donation a été effectuée. Rappelons seulement que Piette accepta de donner sa collection au musée dans une lettre datée du 21 juin 1902, mais que l'acte ne fut signé devant notaire que le 25 février 1904 et accepté par décret du président de la République le 9 juin 1904 alors que la collection était entrée au musée dès le mois d'août 1902. Son intransigeance au sujet de la présentation des objets dans les vitrines engendra de nombreux conflits de 1902 à 1904 (Chollot 1964: 24-25). II obtint finalement de pouvoir venir reclasser lui-même les objets, ce qu'il fit à plusieurs reprises mais ne put achever en raison de problèmes de santé. Devenu de plus en plus ombrageux à la fin de sa vie, il avait des crises de véritable paranoïa où il croyait que le jeune Breuil, pourtant son disciple, voulait le dépouiller de ses collections et de ses travaux (Hurel 2011: 144-145). fig. 4

Exemple de lignée d'outils proposée par A. L. F. PittRivers, in Lane Fox 1868 , repris dans Pitt-Rivers 1906, pl. XV.

fig. 5

Évolution radiale d'armes australiennes à partir d'un simple bâton droit in Lane Fox $1875 a$, repris dans Pitt-Rivers 1906, pl. III. 


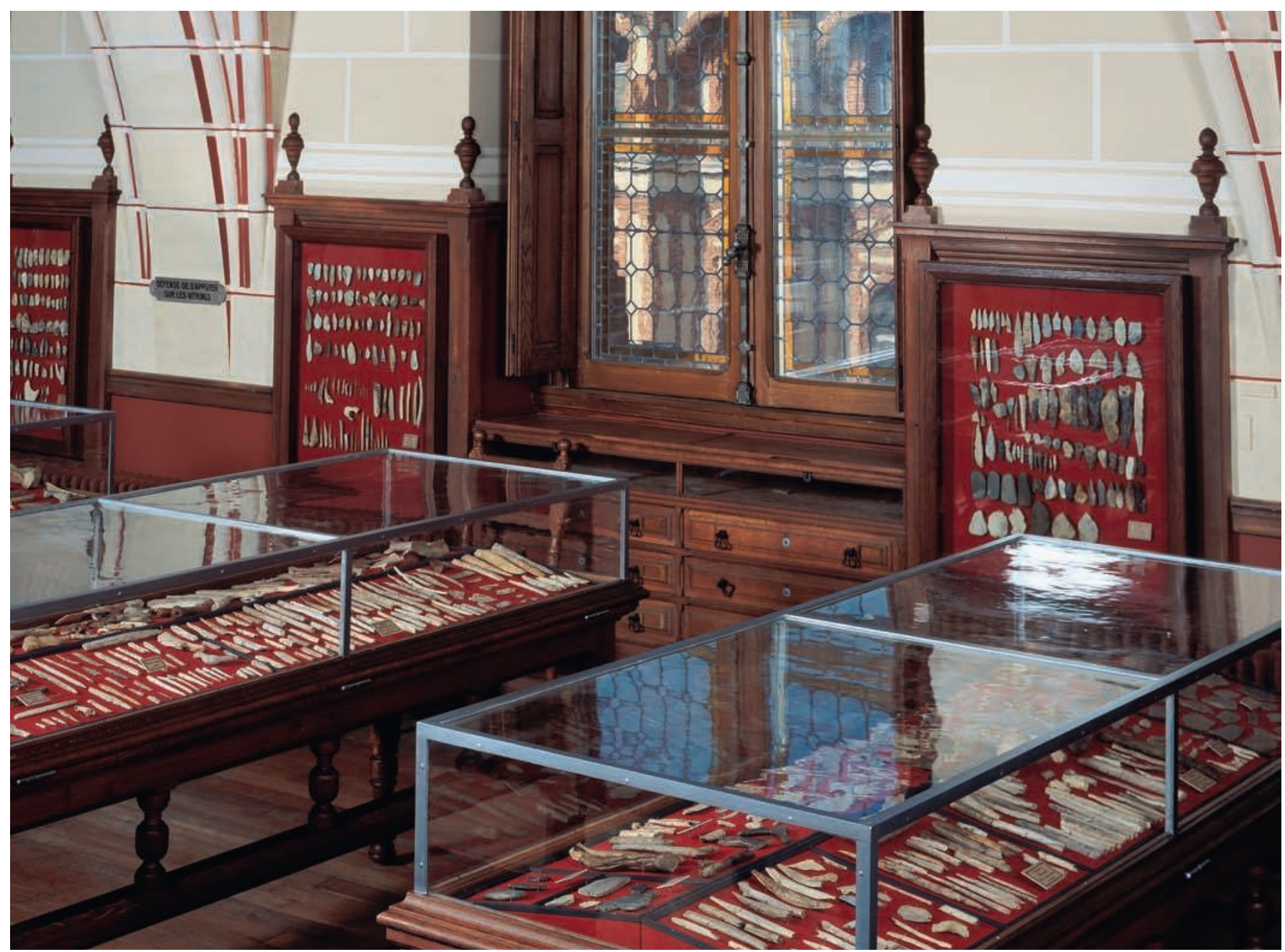

fig. 6

La salle Piette au Musée

d'Archéologie nationale,

fin 2008. (C) RMN-Grand

Palais (musée d'Archéologie

nationale) / Loïc Hamon. 


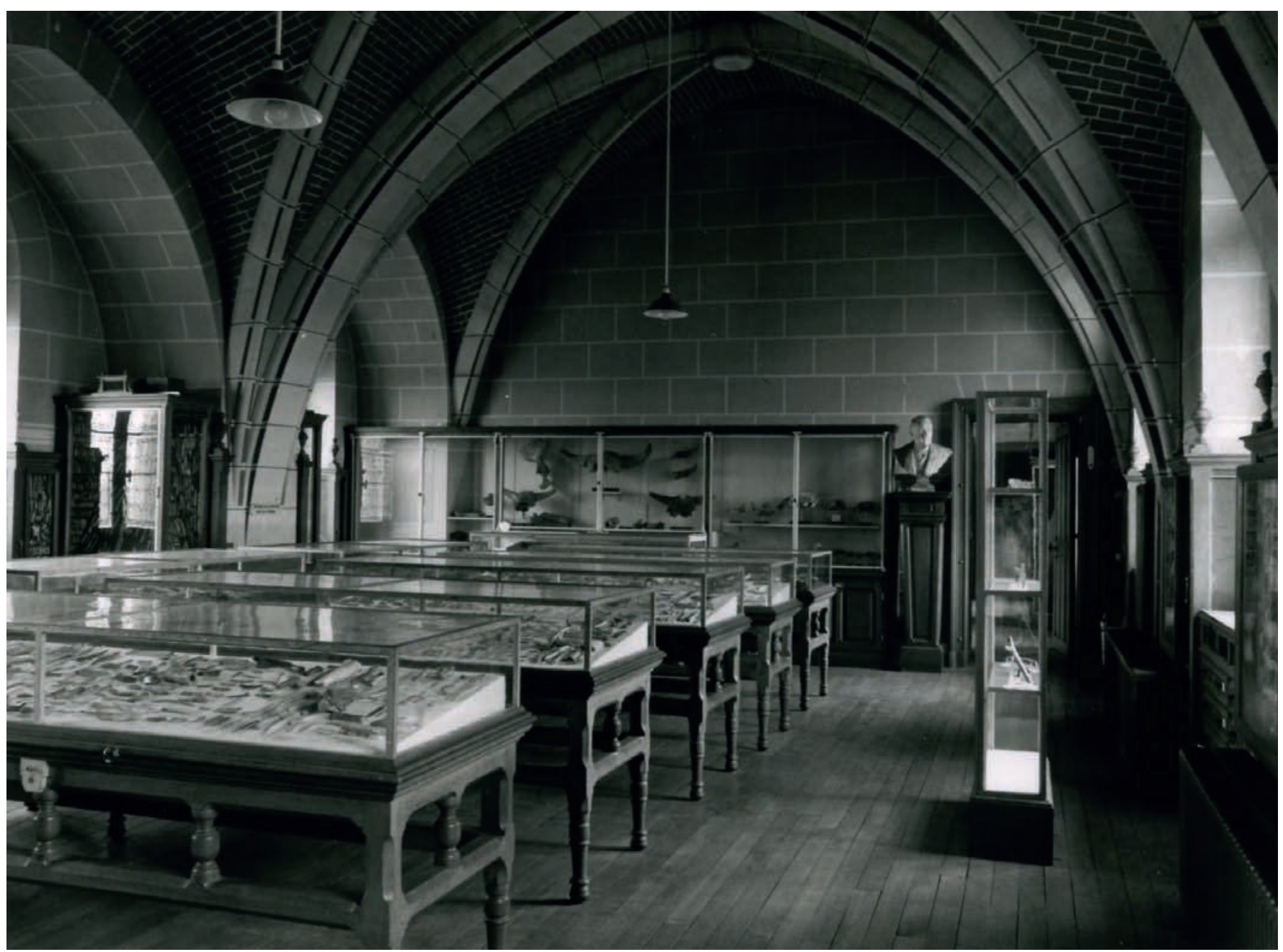

fig. 7

La salle Piette au Musée d'Archéologie nationale.

Cliché postérieur

au remaniement de $\mathrm{H}$. Breuil et M. Chollot, v. 1957.

La rangée de vitrines à fond rouge, non visible sur la photo, se trouve à l'arrièreplan, le long des fenêtres. (C) archives MAN/Charles Hurault. 
22. Une restructuration complète de la salle avait été décidée en 1961, mais la redécouverte, in extremis, de l'acte de donation de Piette a heureusement empêché l'exécution de ce projet (Schwab, communication personnelle).

\section{ci-contre}

fig. 8 a

Vitrine d'art mobilier de la grotte de Lortet sur fond blanc, remaniée par $\mathrm{H}$. Breuil et M. Chollot en 1957, cliché de 2005. (C) Musée d'Archéologie nationale/ Philippe Catro.

\section{ci-contre}

fig. $8 \mathrm{~b}$

La même vitrine après restauration, cliché de fin 2008. Salle Piette, MAN. (C) RMN-Grand Palais (musée d'Archéologie nationale) / Loïc Hamon.
II mourut le 5 juin 1906, avant que la collection soit définitivement installée dans la salle, et c'est Breuil qui poursuivit l'installation, sans l'achever, d'une bonne partie des vitrines à la demande du conservateur du musée, Salomon Reinach, et avec l'aide d'Henri Fischer, gendre et collaborateur du défunt (Breuil 1956: 339; Chollot 1964: 32). Si l'on a peu de détails sur ce travail, Breuil précise toutefois qu'il a respecté les volontés de Piette et n'a fait subir à sa classification que les modifications qu'il jugeait indispensables. En accord avec la famille, il s'est contenté de mentionner la classification du donateur par un tableau mural (Chollot 1964: 30).

Les œuvres d'art mobilier les plus prestigieuses du musée des Antiquités nationales furent mises à l'abri pendant la Première puis la Seconde Guerre mondiale, où elles furent transférées aux châteaux de Valençay et de Chambord (Schwab 2008: 18) comme d'autres chefs-d'œuvre des musées parisiens. Elles «revinrent à leurs places exactes avec quelques dégâts de détail, car il s'agissait de pièces très fragiles » (Breuil 1961: 12).

En 1957, Breuil est à nouveau sollicité par André Varagnac, alors conservateur du musée, pour achever l'aménagement de la salle Piette. II y consacre plusieurs semaines avec l'aide de Marthe Chollot (ibid.). Dans le préambule du catalogue établi pour l'occasion (Chollot 1964), celle-ci affirme à plusieurs reprises que la classification stratigraphique voulue par le donateur avait perduré jusque-là. En réalité, nous verrons que ce n'était pas exactement le cas. Toujours est-il que cet aménagement donne lieu à de profonds remaniements. L'ajout de deux nouvelles vitrines permet d'«aérer» les vitrines existantes (ibid.: 32). De plus et surtout, Breuil et Chollot choisissent de présenter les objets par site. Chollot se justifie en disant que le nouveau rangement «permettra désormais des études sur les caractères régionaux et locaux de l'art mobilier quaternaire" (ibid.). Elle précise cependant qu'elle a scrupuleusement respecté pour chaque site le rangement par couche stratigraphique. La salle rénovée est inaugurée en 1961, en même temps que les salles publiques dont le réaménagement avait été voulu par André Malraux ${ }^{22}$. Des photographies datant de la fin des années 1950 donnent une idée des remaniements opérés. La salle y apparaît comme coupée en deux, avec d'un côté les vitrines les plus modifiées, reconnaissables à leur fond blanc, de l'autre les vitrines à fond rouge, sans doute peu transformées (fig. 7). Outre leur fond blanc, les vitrines "aérées" se distinguent par la moindre densité des objets exposés (en moyenne 200 contre 700 pour celles à fond rouge), qui y sont simplement posés sans être fixés sur des socles métalliques (fig. 8a). On a peu de détails sur les remaniements effectués ensuite. On sait seulement que les vitrines contenant le matériel de Brassempouy ont été entièrement revues par Delporte dans les années 1980, époque où il a repris la fouille du site.

En 2005, après cent ans d'existence, la salle avait vieilli, les fonds textiles étaient défraîchis, les baguettes de séparation en mauvais état, les cartels manuscrits effacés. Par ailleurs, les systèmes d'accrochage métalliques étaient agressifs pour les objets. Une restauration, indispensable ne serait-ce que pour sauvegarder les pièces qui risquaient de s'abîmer, a donc été réalisée de 2005 à 2008 par l'actuelle équipe du département de préhistoire du musée. II s'agissait de remettre la salle dans son état originel 

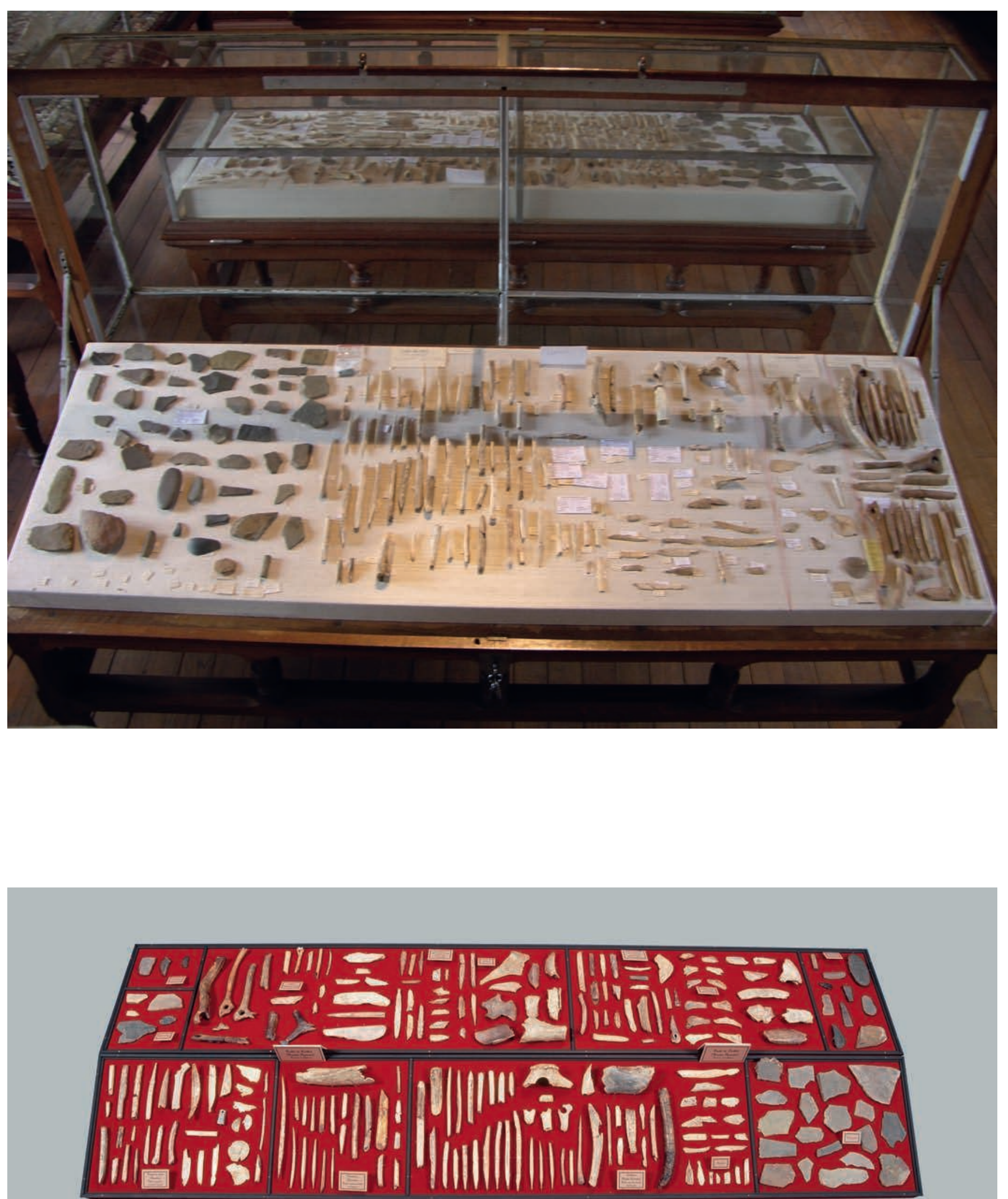


\section{ci-contre}

fig. 9 a, b et c

Vitrine de l'industrie lithique et osseuse de la grotte

de Lortet. Salle Piette, Musée d'Archéologie nationale.

a. cliché v. 1957, postérieur

au remaniement de $\mathrm{H}$. Breuil et M. Chollot.

b. cliché de 2005, antérieur

à la restauration.

c. cliché de fin 2008,

postérieur à la restauration.

C Musée d'Archéologie

nationale/Loïc Hamon. afin d'en faire une sorte de musée dans le musée tout en respectant les clauses de la donation. D'après Catherine Schwab, un compromis a été trouvé entre la présentation ancienne - qui ne pouvait être modifiée - et les normes actuelles de sécurité et de conservation.

Un travail de récolement et d'inventaire a été réalisé vitrine par vitrine en utilisant les archives photographiques, dont les clichés datent pour la plupart de la fin des années 1950. II a été décidé de ne pas conserver l'organisation des vitrines à fond blanc, remaniées par Breuil et Chollot, et de tenter de leur rendre sinon la disposition voulue par Piette, du moins l'esprit de sa présentation (fig. 8b). On a tenu pour allant de soi que les huit vitrines à fond rouge étaient conformes à la disposition de 1904, hormis éventuellement quelques pièces sorties pour étude ou pour une exposition et replacées différemment dans la vitrine. Cette présupposition est surprenante car les objets y sont classés par site (fig. 9a, b et c) mais, pour au moins trois d'entre elles, on a des raisons de la croire fondée. Il s'agit de celles présentant respectivement les objets de Gourdan, de Lortet et d'Arudy. Indépendamment du fond rouge, la densité des objets indique en effet qu'elles sont antérieures à la réorganisation de Breuil et Chollot. On peut supposer soit que ces trois vitrines ont été composées par l'abbé Breuil juste après la mort de Piette et que c'est lui qui a choisi la présentation de l'outillage par site; soit que c'est Piette lui-même, décidant de faire une exception à sa propre règle, qui a décidé de consacrer une vitrine à chacun des trois sites, peut-être en raison du grand nombre d'objets qu'ils avaient livrés. Cette dernière hypothèse me paraît être la plus vraisemblable, car on voit sur des photographies de ces vitrines datant de 2005 (fig. 9b) - donc antérieures à la restauration - que les pièces y étaient soclées, c'est-à-dire fixées par des griffes en laiton, ce qui indique que Piette en avait validé la position. En effet, les objets installés provisoirement, en attente de sa validation, étaient simplement fixés par des clous et certaines vitrines présentaient encore de tels arrangements provisoires avant 2005.

Malgré le soin apporté à la restauration de 2005-2008, on voit que, excepté le cas de ces trois vitrines pour lesquelles un doute subsiste, la présentation n'a pas été conforme aux vœux de Piette. II n'y a évidemment pas de raisons d'en faire le reproche à l'équipe de la restauration, qui s'est de bonne foi voulue fidèle à l'esprit de Piette. De fait, la salle actuelle restitue fort bien l'idée que nous pouvons nous faire aujourd'hui de l'esprit d'un savant de la fin du $x x^{e}$ siècle. Dernière précision: depuis le 29 novembre 2008, avec un siècle de retard, la salle est ouverte au public par petits groupes. Cette clause de l'acte de donation n'avait jamais été respectée.

\section{Genèse et destin du Pitt-Rivers Museum}

N'ayant subi que peu de modifications depuis l'ère victorienne, le Pitt-Rivers Museum est considéré aujourd'hui comme un musée de musée. Les objets y sont classés par type et par fonction et non par aire culturelle. Les vitrines sont pleines à craquer d'objets quotidiens de toutes époques venus du monde entier (fig. 10). Le musée a été fondé en 1884 pour abriter la collection d'objets archéologiques, ethnologiques et d'antiquités donnée à I'université par Pitt-Rivers. II a ouvert officiellement ses portes en 1891, mais 

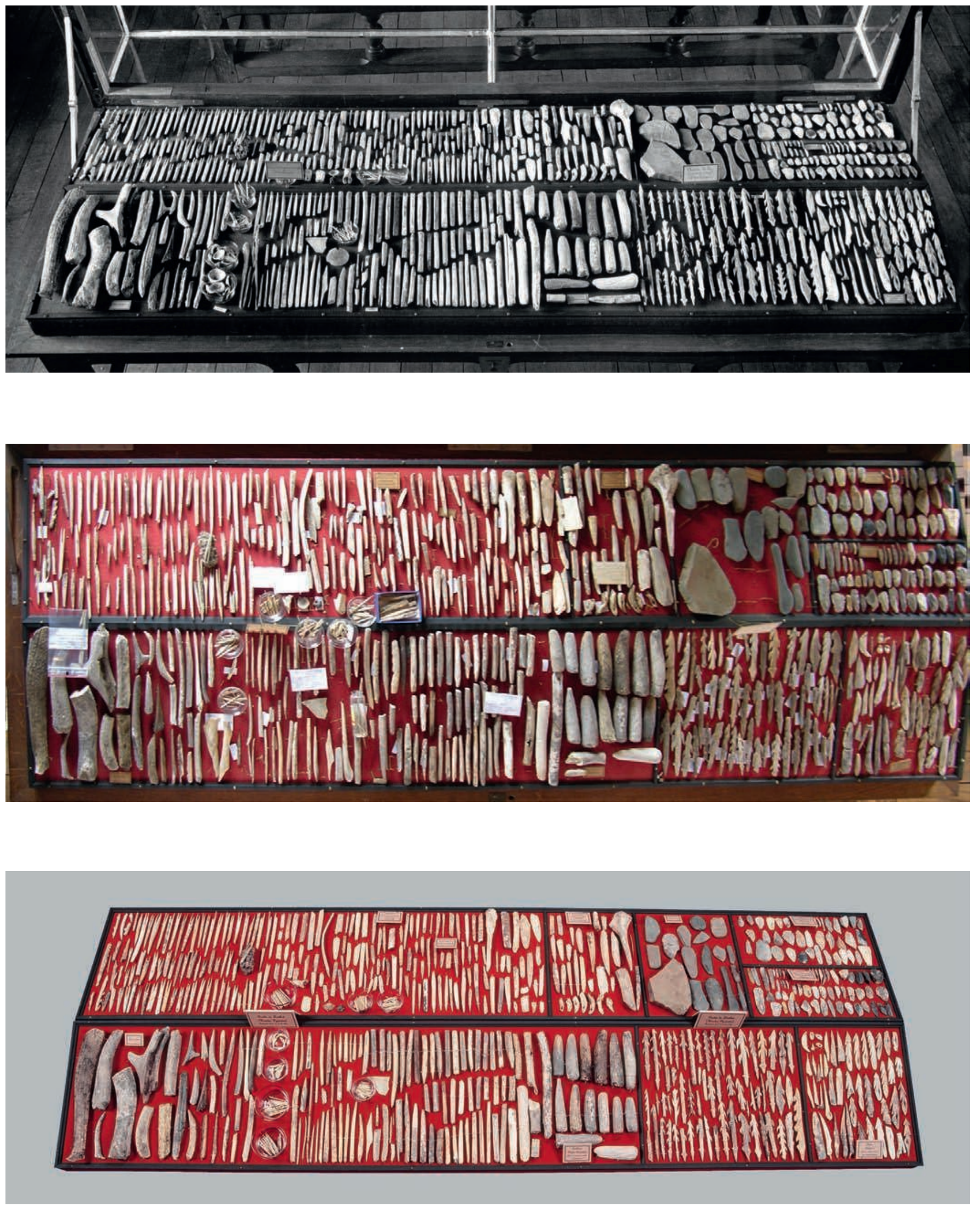

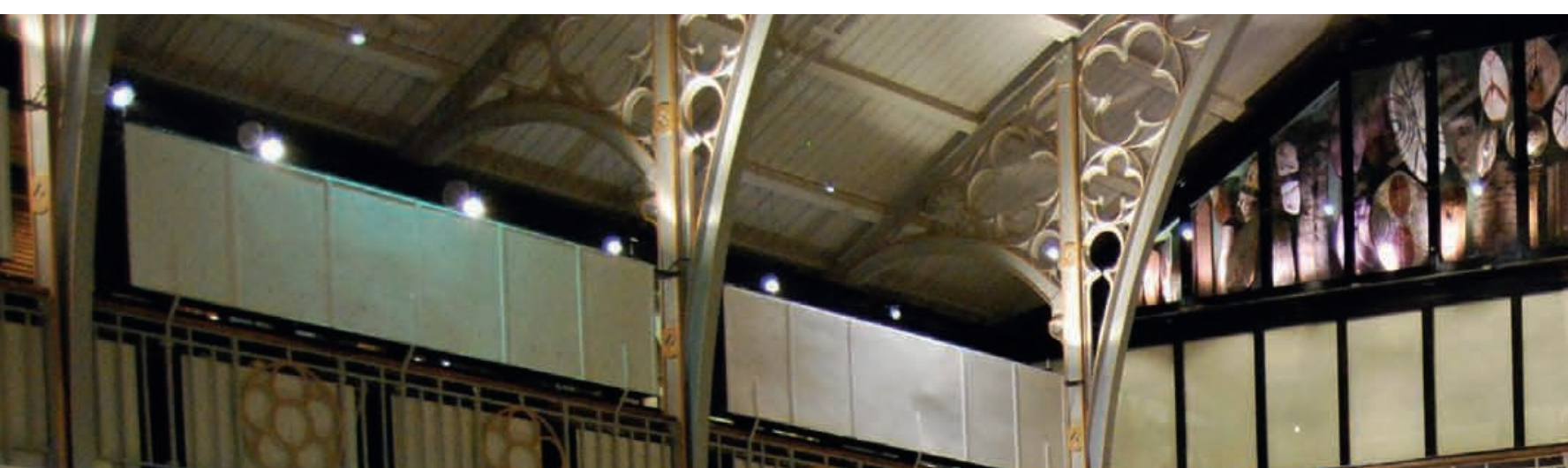

3 (ritionso
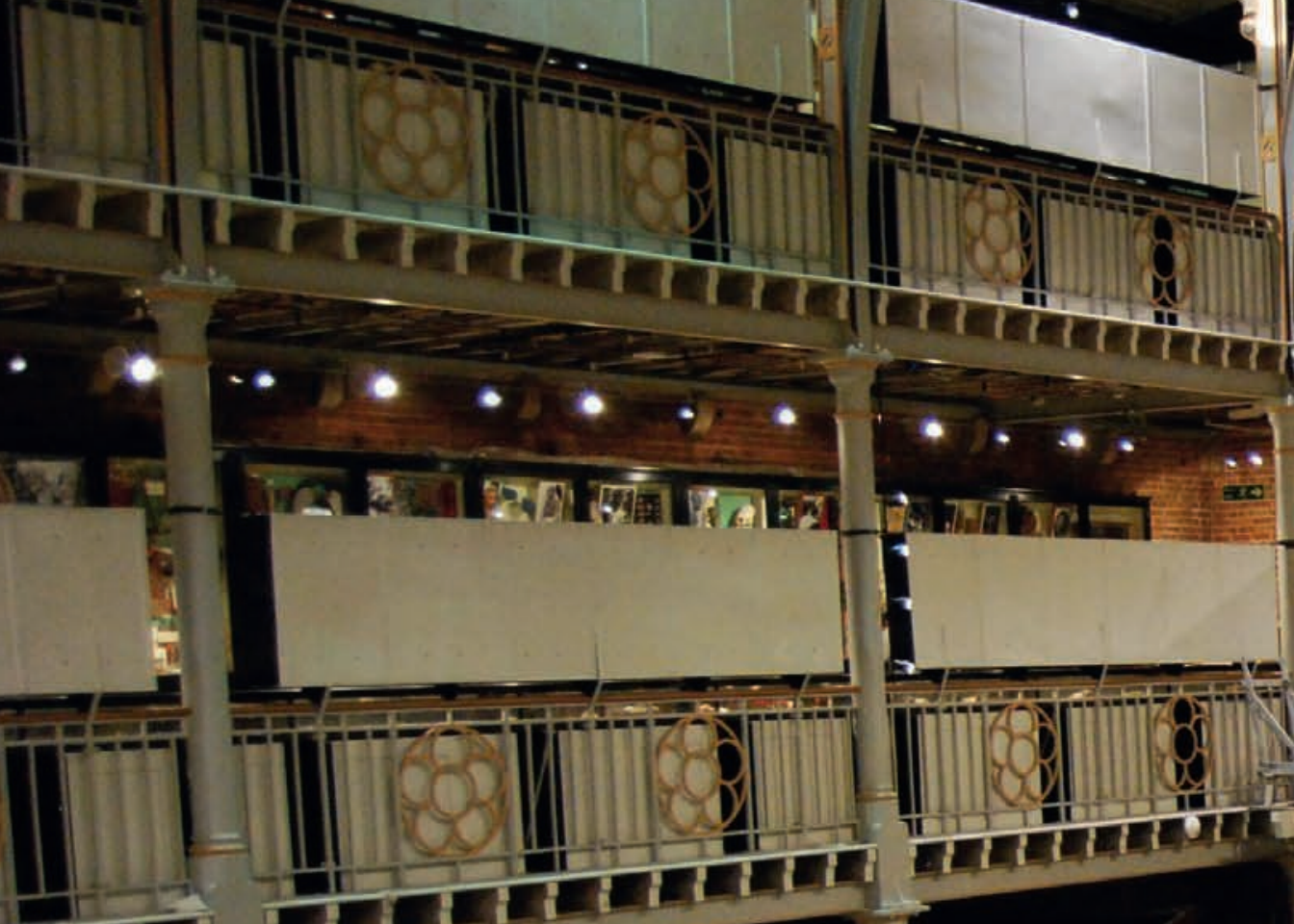

(5)

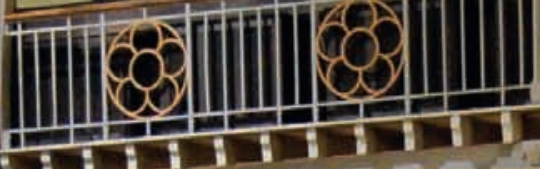

; i i i I I I
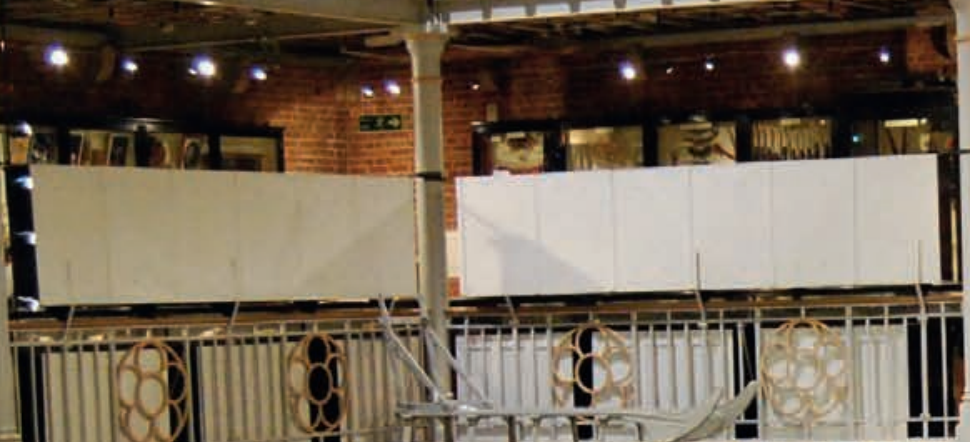

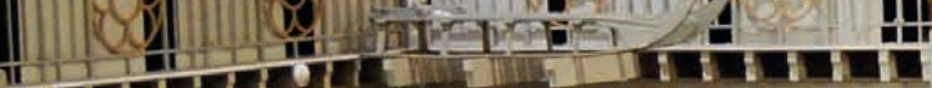
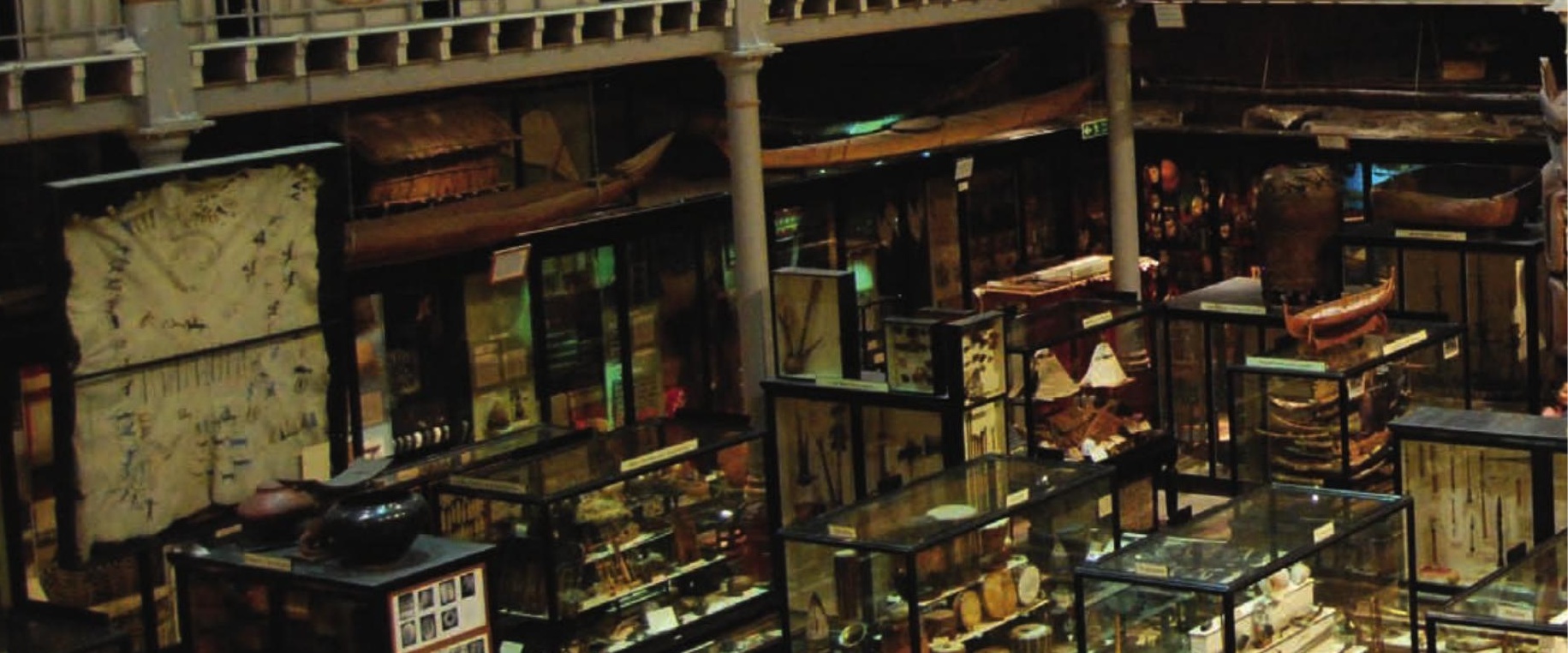


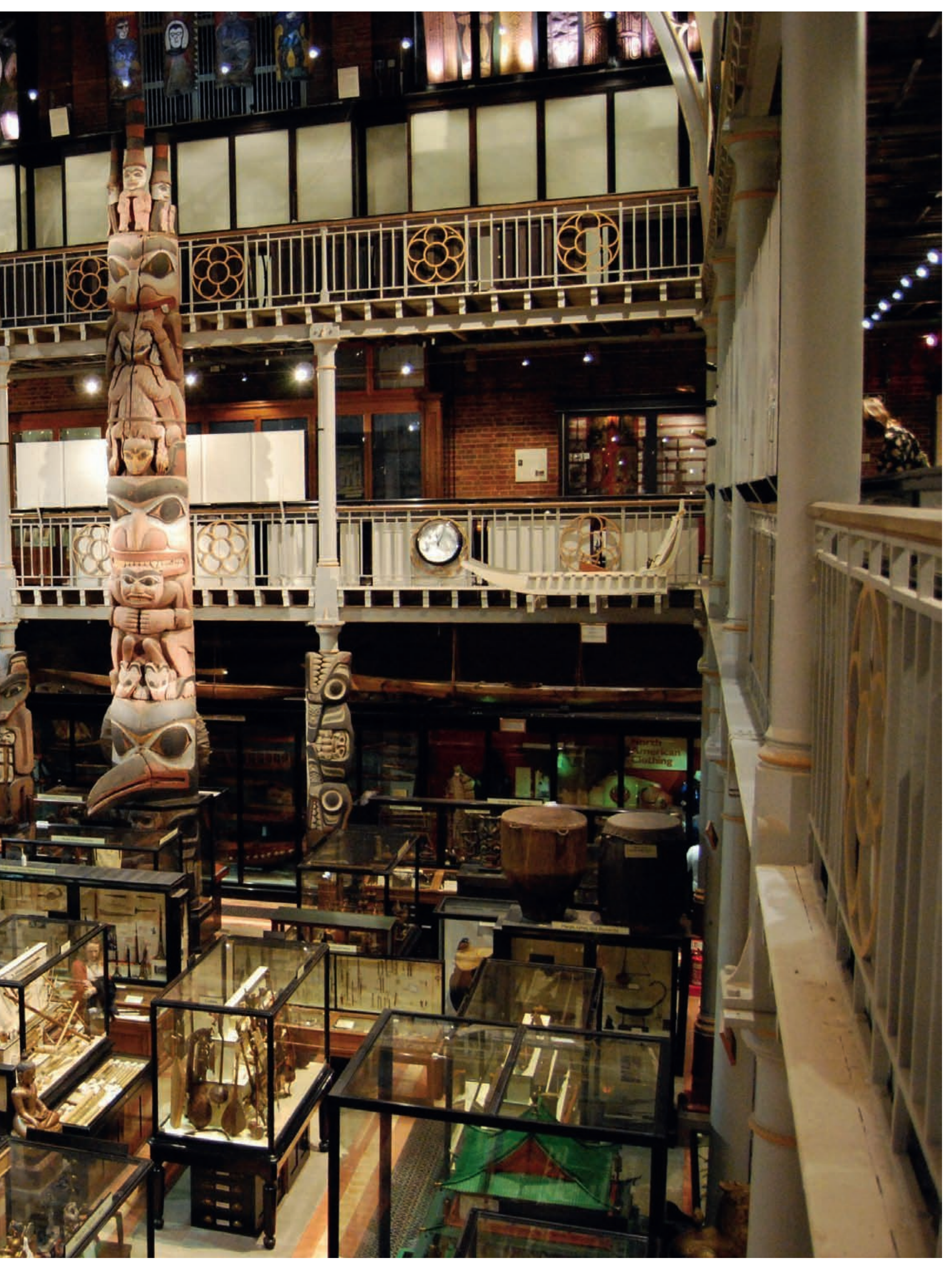


23. http://web.prm.ox.ac uk/rpr, Articles, C. Founding Collection, Introduction.

24. http://web.prm.ox.ac. uk/rpr, Articles, D. Second Collection, Introduction.

double page

fig. 10

Le Pitt-Rivers Museum

aujourd'hui. () Jeremy Macclancy. Avec l'autorisation du Pitt-Rivers Museum, University of Oxford. les objets ont commencé à y arriver à la fin de l'été 1885. Nous n'avons que peu de détails sur les opérations de transfert depuis le South Kensington Museum à Londres, si ce n'est que certaines vitrines verticales en bois sur lesquelles étaient fixées des armes ont aussi été transportées, mais on ignore si les objets ont ensuite été replacés à l'identique sur les panneaux ${ }^{\mathbf{2 3}}$.

La collection a cependant été enrichie, dès les premières années, par l'ajout de milliers d'objets similaires provenant du Muséum d'histoire naturelle d'Oxford et de l'Ashmolean Museum. Leur transfert a été effectué par le premier conservateur, Henry Balfour, et ses successeurs ont continué à alimenter considérablement les collections. Très vite donc, le musée n'était plus exclusivement constitué des collections de Pitt-Rivers. Il compte aujourd'hui 300000 objets et autant de photographies de terrain, ainsi que des collections importantes de manuscrits et d'enregistrements (Coote 2011), de sorte que la collection Pitt-Rivers ne représente plus que 7,5\% de l'ensemble.

Après sa donation à Oxford, Pitt-Rivers a continué à collecter des objets de 1880 à 1900. Cette seconde collection - évaluée à environ 30000 objets - a été exposée dans un nouveau musée qu'il créa à Farnham, dans le Dorset, à quelque 3 miles de sa propriété de Rushmore Lodge, avant d'être vendue par ses descendants dans les années 1960 et dispersée dans des centaines de collections publiques et privées du monde entier ${ }^{24}$. PittRivers s'est désintéressé très vite du sort des objets conservés à Oxford et c'est Balfour qui s'est occupé de l'installation des premières vitrines. S'il suivit les idées du général à propos de l'évolution des formes du plus simple au plus complexe, ce dernier avait ses propres théories sur l'évolution et il tenait en particulier beaucoup à la notion d'hybridation sur laquelle il a du reste publié (Balfour 1893). Les lignées de Pitt-Rivers divergent à partir d'une origine commune; son modèle présente la forme d'une étoile dont les branches sont susceptibles de se développer dans des ramifications infinies puisque de nombreuses évolutions sont possibles à partir d'une même forme de base. Les lignées de Balfour résultent au contraire du croisement entre deux lignées de la même manière que le croisement entre deux individus d'une même espèce permet d'obtenir des hybrides. Les objets étaient bien, dès l'origine du musée, arrangés en séries typologiques afin d'illustrer le développement des techniques, des formes et des idées, mais la façon de les exposer était davantage conforme aux conceptions de Balfour qu'à celles de Pitt-Rivers (Coote 2011) (fig. 11).

Dès 1940, après la mort de Balfour survenue en 1939, et pour répondre aux changements intervenus en anthropologie et en archéologie, les séries évolutionnistes furent démantelées. Toujours organisées par types - toutes les lances ensemble, tous les paniers, tous les jouets... -, les vitrines furent arrangées de façon à illustrer la variabilité des solutions que les différentes populations du monde ont imaginées pour résoudre des problèmes identiques: comment se chauffer (équipement pour produire du feu, vêtements...), comment cuisiner, stocker et transporter la nourriture (pots, vaisselles, paniers...), comment conjurer le sort (amulettes, charmes...), etc. (ibid.). Jeremy Coote nous a signalé la seule vitrine considérée aujourd'hui comme conforme à l'ancien esprit. II s'agit d'un essai de reconstitution réalisé dans les années 1950 avec une série de peignes acquis en 1951 qui 
ne nous a pas paru très convaincant. Les vitrines d'armes - et en particulier d'armes à feu - sont sans doute les plus proches de ce qu'avait conçu Pitt-Rivers et celles qui auraient subi le moins de transfor-mations (Coote, communication personnelle) (fig. 12).

Depuis les années 1980, les conservateurs ont pris conscience des qualités esthétiques indéniables du musée, et un très gros effort a été fait pour maintenir et restituer l'aspect général des vitrines d'époque victorienne (fig. 13). Cela n'a pas empêché la modernisation avec, en particulier, la mise à disposition du public, depuis 2000, d'une énorme documentation sur internet ${ }^{26}$ (Coote 2011).

L'une des questions auxquelles s'est attelée l'équipe de recherche du musée concerne la façon dont Pitt-Rivers avait conçu ses vitrines. Elle tente d'y répondre à partir de l'exploration des catalogues et inventaires, de la correspondance et des conférences transcrites de Pitt-Rivers. Deux autres pistes sont suivies. La première concerne l'organisation des collections avant leur arrivée à Oxford ${ }^{26}$. Dès 1877, Pitt-Rivers avait réalisé un catalogue de ses objets, alors présentés au South Kensington Museum à Londres. Mais celui-ci est incomplet, et seules les parties I et II, consacrées essentiellement aux armes, ont été publiées (Lane Fox 1877). Quelques inventaires accompagnaient également les objets transférés ${ }^{\mathbf{2 7}}$. Mais aucun de ces documents ne nous renseigne sur la disposition des objets dans les vitrines du South Kensington Museum et on n'en connaît nulle photographie (si tant est qu'il en ait existé). La seconde piste concerne la «seconde collection", exposée au musée de Farnham et soigneusement inventoriée par PittRivers lui-même avant d'être dispersée par ses descendants. Ce magnifique catalogue manuscrit en neuf volumes très illustrés ${ }^{28}$, riche d'informations sur les vendeurs, les enchères et le prix des objets, est une source précieuse sur l'histoire de la collection et a même permis d'identifier certaines pièces ${ }^{29}$. Mais il est là encore de peu d'utilité pour savoir comment étaient disposés les objets dans les vitrines du musée de Farnham ${ }^{30}$.

\section{Conclusion}

Que ce soit à Oxford ou dans le château de Saint-Germain-en-Laye, force est de constater que, malgré la volonté des donateurs, l'agencement des collections ne refléta sans doute jamais leur théorie scientifique, ou pour bien peu de temps. Balfour à Oxford ou l'abbé Breuil à Saint-Germain-en-Laye prirent très vite les choses en main et donnèrent à la présentation des collections leurs touches personnelles. Peut-on parler de véritables trahisons?

Les préoccupations chronologiques de Piette correspondaient à un état de la discipline et, s'il a tenté d'y apporter des réponses les plus précises possibles, il n'a fait que pousser de manière maniaque et quasi obsessionnelle des démarches qui étaient les mêmes que celles de ses collègues préhistoriens. Breuil n'a donc fait, en toute bonne conscience, qu'actualiser, en gardant le même esprit général, les connaissances chronologiques qui avaient évolué depuis l'époque de Piette. Quant à la réorganisation des vitrines par site, on peut supposer à sa décharge qu'il n'a pas mesuré l'importance que Piette donnait à la chose. En revanche, les questionnements de Pitt-Rivers, véritable cognitiviste avant l'heure, sur
25. www.prm.ox.ac.uk.

26. http://web.prm.ox.ac uk/rpr, Articles, B.7.1.2 Comparing London and Oxford Series.

27. Appelés le «Black book», le «Red book», le «Blue book", etc., selon la couleur de leur reliure; http://web. prm.ox.ac.uk/rpr, Articles, B.7.2.2.a Introduction to the Black Book.

28. http://web.prm.ox.ac.uk/ rpr, Databases et http://janus. lib.cam.ac.uk, MS Add.9455.

29. http://web.prm.ox.ac. uk/rpr, Articles, D.1.1 An Introduction to the Catalogue.

30. Offert par la famille du petit-fils du général à l'université de Cambridge, où il est conservé dans les collections du département des manuscrits de la bibliothèque, ce catalogue est maintenant tombé dans le domaine public et peut faire l'objet d'études. 


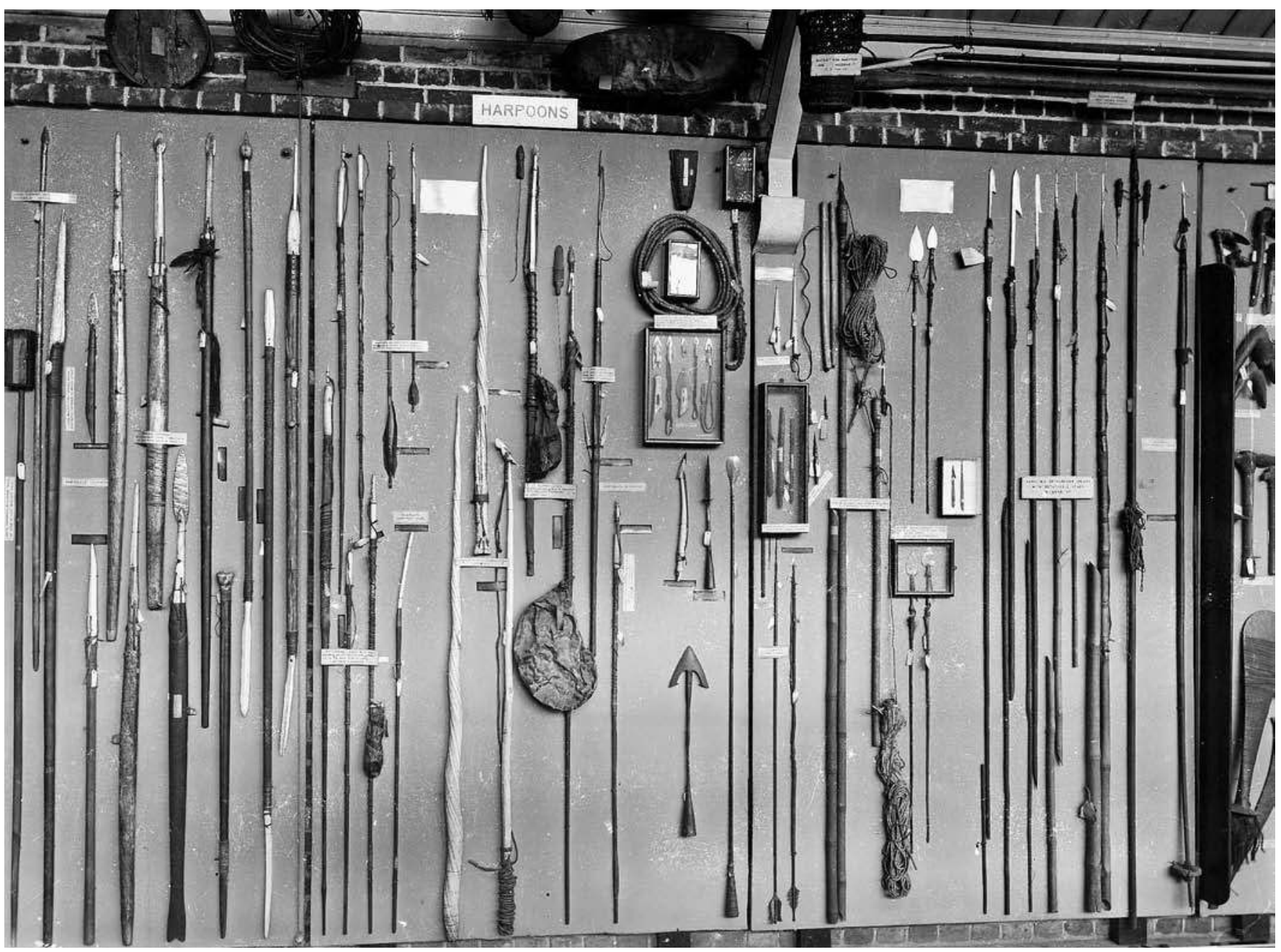

fig. 11

Panneau de harpons dans la galerie supérieure du

Pitt-Rivers Museum entre

1900 et 1910 , cliché Alfred

Robinson. (c) Pitt-Rivers

Museum, University of Oxford. 

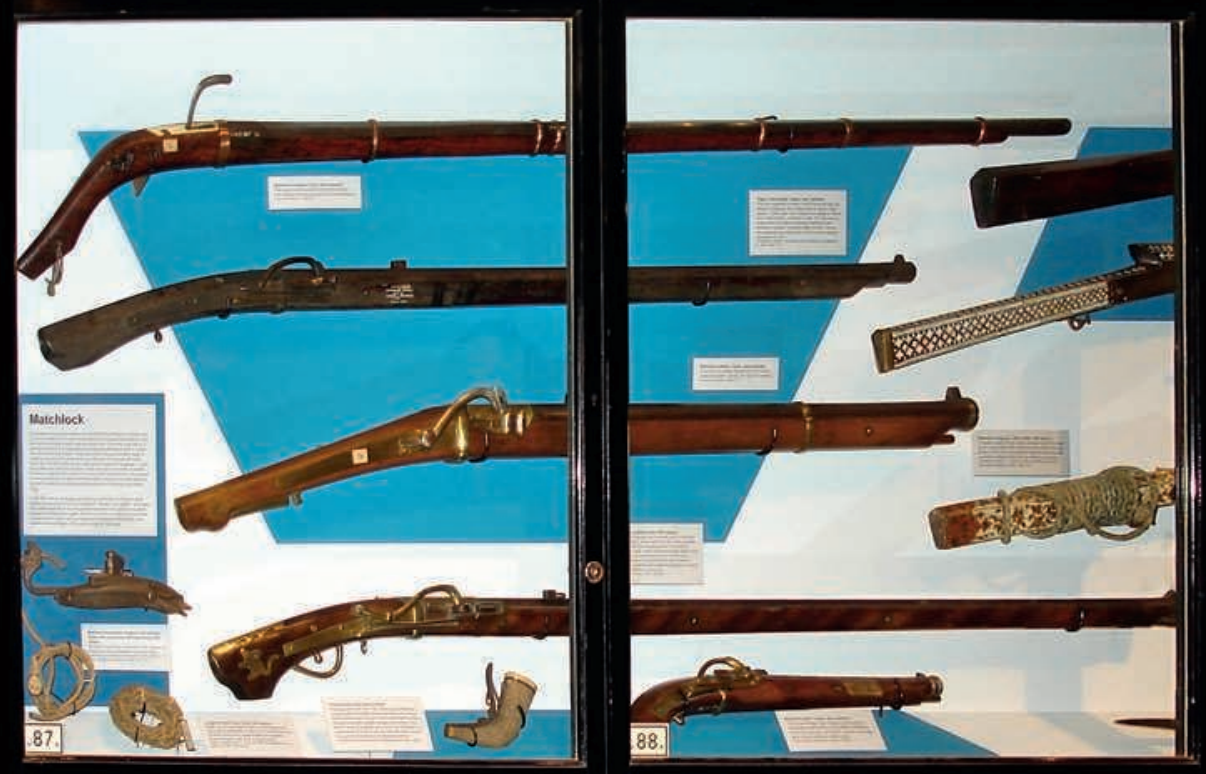

fig. 12

La première de la série

de vitrines d'armes à feu

du Pitt-Rivers Museum.

(C) Sophie A. de Beaune, avec

l'autorisation du Pitt-Rivers

Museum, University of Oxford. 


\section{remerciements}

Je remercie Jeremy Coote pour son accueil au Pitt-Rivers Museum. Un grand merci aussi à Catherine Schwab, conservatrice au musée d'Archéologie nationale, qui a mis à ma disposition clichés et archives du musée. Je tiens également à exprimer ma reconnaissance aux membres de l'atelier "Manuscrits en cours » du Centre d'études des mondes africains pour leur remarques, qui ont contribué à l'amélioration de ce texte. l'évolution de l'inventivité humaine se démarquaient de ceux de ses contemporains. II se trouve que Balfour avait lui aussi des idées sur la question et qu'il a mis en scène sa propre théorie pour organiser les vitrines. Et il l'a fait avec d'autant moins de scrupules que Pitt-Rivers s'était désintéressé de son vivant de la collection conservée à Oxford et avait lui-même autorisé les changements s'ils étaient conformes à l'évolution de la science.

Pour finir, notons que les deux hommes ne semblent pas s'être connus. La rencontre ne s'est faite qu'à la génération suivante, avec la lecture, par le jeune abbé Breuil, de la publication de Balfour sur l'évolution de l'art décoratif (Balfour 1893). L'idée d'une dégénérescence de l'art, que Breuil a développée dans sa thèse d'habilitation pour Fribourg en 1904, lui serait venue après la lecture de cet ouvrage. En 1912, il rencontra même Balfour qui lui fit les honneurs du Pitt-Rivers Museum (Breuil, autobiographie inédite, chap. x, cité par Hurel 2011: 141 et 147). Cette lecture intervint peu avant le moment où Breuil s'est occupé de l'installation de la collection de Piette au musée de Saint-Germain-en-Laye.

Piette et Pitt-Rivers, ces deux savants imprégnés des idées nouvelles de leur temps, à l'époque où la préhistoire et l'anthropologie sociale étaient encore balbutiantes, suivirent des chemins parallèles, sans se croiser et sans que leurs idées soient reprises par d'autres. Ils avaient pour ambition d'utiliser leurs collections pour convaincre de la justesse de leurs idées, mais, si leurs musées sont en effet passés à la postérité, le message qu'ils étaient supposés transmettre est tombé dans l'oubli. Ironie du sort pour des hommes qui avaient voué leurs recherches à l'évolution et au progrès des connaissances de l'humanité. L'idée que les objets d'une même aire culturelle forment système s'est imposée dès les premières décennies du $\mathrm{xx}^{\mathrm{e}}$ siècle et a orienté par la suite l'organisation de tous les grands musées de civilisation et d'ethnologie (Descola 2007: 140). Du côté des musées archéologiques, les modèles auront été plus variés, certains privilégiant la présentation des belles pièces, à l'instar des musées des beaux-arts, d'autres cherchant à resituer les vestiges de manière synchronique et à leur redonner vie. Quant à la présentation chronologique souhaitée par Piette, elle a aujourd'hui perdu son intérêt puisque le problème de la datation a été résolu de tout autre manière.

L'ambition de garder telles quelles des collections sans modification jusqu'à la nuit des temps était une de ces utopies comme sut en engendrer le $x \mid x^{e}$ siècle finissant. Elle était ferme et têtue chez Piette, plus souple et plus amène chez Pitt-Rivers. Leurs collections devaient illustrer leurs idées, et ceux qui en ont reçu le dépôt, fidèles dans leur infidélité, les ont réorganisées à la lumière de leurs propres idées. Sans cette infidélité, elles seraient sans doute aujourd'hui ensevelies sous la poussière qui a recouvert bien d'autres musées. Elle aura donc permis que quelque chose perdure de l'esprit des donateurs. 


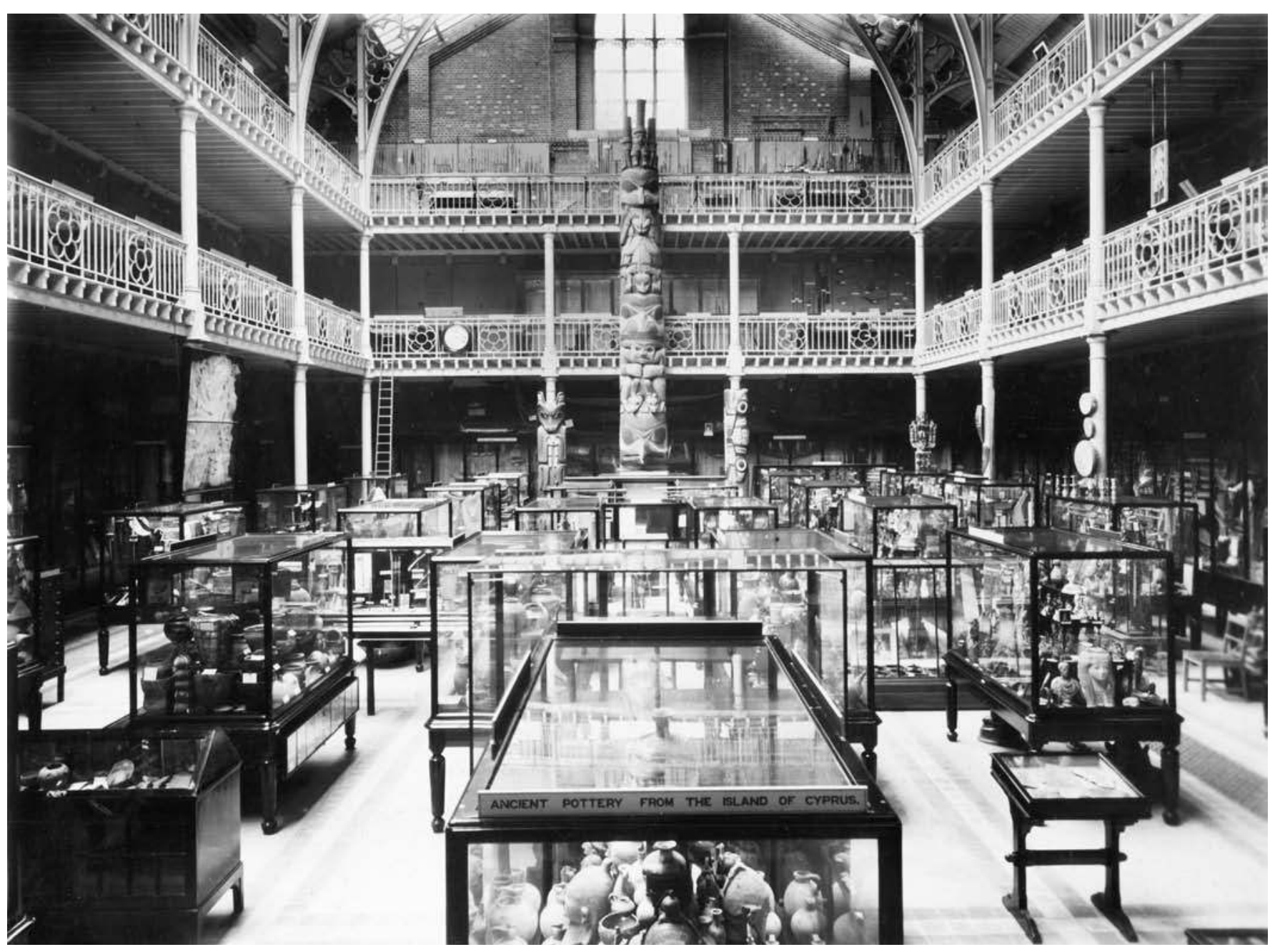

fig. 13

Vue du Pitt Rivers Museum

Court, vers l'aile est, près

des objets haida (photographie

acquise en 1901), cliché

Alfred Robinson (c) Pitt-Rivers

Museum, University of Oxford. 


\section{Balfour, Henry}

1893 The Evolution of Decorative Art. Londres, Rivington, Percival \& Co.

\section{Bowden, Mark}

1991 Pitt-Rivers. The Life and Archaeological Work of LieutenantGeneral Augustus Henry Lane Fox Pitt-Rivers. Cambridge, Cambridge University Press.

\section{Breuil, Henri}

1909 "L'évolution de l'art quaternaire et les travaux d'Édouard Piette ", Revue archéologique 4(13): 378-411.

1956 «Paroles prononcées à la cérémonie commémorative du cinquantenaire du décès d'Édouard Piette à Rumigny (Ardennes), le 14 octobre 1956 ", Bulletin de la Société préhistorique française 53(7-8) : 337-339.

1961 "Préface», in Chollot 1964 : 11-13.

\section{Capitan, Louis}

1900 «Exposition de l'École d'anthropologie et de la souscommission des Monuments mégalithiques. Catalogue raisonné et descriptif ", Bulletins de la Société d'anthropologie de Paris 5(1): 295-319.

\section{Chapman, William Ryan}

1981 Ethnology in the Museum: A.H.L.F. Pitt-Rivers (1827-1900) and the Institutional Foundations of British Anthropology. University of Oxford, thèse de doctorat.

1991 "Like a game of dominoes: Augustus Pitt-Rivers and the typological museum idea ", in Susan Pearce (éd.), Museum Economics and the Community. Londres, The Athlone Press ( New Research in Museum Studies » 2): 135-176.

\section{Chollot, Marthe}

1964 Musée des Antiquités nationales, collection Piette. Paris, Éditions des Musées nationaux.

\section{Cohen, Claudine}

2011 La Méthode de Zadig. La trace, le fossile, la preuve. Paris, Seuil.

\section{Coote, Jeremy}

2011 "Speaking for themselves», Interpretation Journal 16(1); www. ahi.org.uk.

\section{Delporte, Henri}

1987 Piette, Pionnier de la préhistoire. Paris, Picard.

\section{Descola, Philippe}

2007 "Passages de témoins", Le Débat 5(147): 136-153.

\section{Evans, John}

1850 "On the date of British coins ", Numismatic chronicle 12 : 127-137.

1875 «The coinage of the Ancient Britons and natural selection", Notices of the proceedings of the Royal Institution of Great Britain 7: 476-487.

\section{Hurel, Armand}

2011 L'Abbé Breuil. Un préhistorien dans le siècle. Paris, CNRS Éditions.

\section{Lane Fox, Augustus Henry}

1868 "Primitive warfare ॥", Journal of the Royal United Services Institution 12: 399-439.

\section{5a «On the principles of} classification adopted in the arrangement of his anthropological collection, now exhibited in the Bethnal Green Museum ", Journal of the Anthropological Institute 4: 293-308.

$\mathbf{1 8 7 5 b}$ "On the evolution of culture ", Journal of the Royal Institute 7 : 357-389.

\section{Catalogue of the} Anthropological Collection lent by Colonel Lane Fox for Exhibition in the Bethnal Green Branch of the South Kensington Museum, June 1874 Parts I and II. Londres, Science and Art Department of the Committee of Council on Education HMSO.

\section{Piette, Édouard}

1895 «Hiatus et lacune. Vestiges de la période de transition dans la grotte du Mas d'Azil ", Bulletin de la Société d'anthropologie de Paris : 235-267.

1907 L'Art pendant l'âge du Renne. Paris, Masson.

\section{Pitt-Rivers, Augustus Henry Lane Fox}

1888 "Address as President of the Anthropological Section of the British Association, Bath, September 6, 1888 ", Report of the British Association for the Advancement of Science : 825 835; http://web.prm.ox.ac.uk/rpr, Primary Documents, C. Published or Unpublished Sources and sources delivered to the public at lectures.

1889-1890 "On the uses and arrangements of arts museums ", conférence au Blackmore Museum, Salisbury; http://web.prm.ox.ac. uk/rpr, Articles, B.1.1 The Ideal Arrangement of Museums according to Pitt-Rivers.

1891 "Typological museums, as exemplified by the Pitt-Rivers Museum at Oxford, and his provincial museum at Farnham, Dorset ", Journal of the Society of Arts 40: 115-122; http://web. prm.ox.ac.uk/rpr, Articles, B.1.1 The Ideal Arrangement of Museums according to Pitt-Rivers.

1906 The Evolution of Culture and Other Essays, J.L. Myres (éd.). Oxford, Clarendon Press [réédition de sept textes parus entre 1858 et 1875].

\section{Reinach, Salomon}

1906 "Nouvelles archéologiques et correspondance: Édouard Piette " [notice nécrologique], Revue archéologique: 151.

\section{Schlanger, Nathan}

2010 "Series in progress: Antiquities of nature, numismatics and stone implements in the emergence of prehistoric archaeology ", History of Science 48(3-4) : 343-370.

\section{Schwab, Catherine}

2008 "La réouverture de la salle Piette: histoire d'une restauration ", Antiquités nationales 39: 9-24.

\section{Severi, Carlo}

2003 "Warburg anthropologue ou le déchiffrement d'une utopie. De la biologie des images à l'anthropologie de la mémoire ", L'Homme 165(1) : 77-128.

\section{Sigaut, François}

1990 "De la technologie à l'évolutionnisme, l'œuvre de Pitt-Rivers », Gradhiva 8: 20-37.

\section{Spencer, Herbert}

1855 Principles of Psychology. Londres, Longman, Brown, Gren and Longmans.

\section{Thompson, Michael W.}

1977 General Pitt-Rivers. Evolution and Archaeology in the Nineteenth Century. Bradford-on-Avon, Moonraker.

\section{Thompson, Michael et Renfrew, Colin}

1999 "The catalogues of the Pitt-Rivers Museum, Farnham, Dorset ", Antiquity 77(280): 377-393. 
